\title{
Low-thrust tour of the main belt asteroids
}

\author{
Marilena Di Carlo* \\ Department of Mechanical and Aerospace Engineering, University of Strathclyde, 75 \\ Montrose Street, G1 1XJ, Glasgow, United Kingdom. \\ Massimiliano Vasile* \\ Department of Mechanical and Aerospace Engineering, University of Strathclyde, 75 \\ Montrose Street, G1 1XJ, Glasgow, United Kingdom. \\ Jamie Dunlop* \\ Department of Mechanical and Aerospace Engineering, University of Strathclyde, 75 \\ Montrose Street, G1 1XJ, Glasgow, United Kingdom.
}

\begin{abstract}
This work presents some initial results on a possible low-thrust tour of the main asteroid belt. The asteroids are visited through a series of fly-by's to be completed within a given time-frame and limit on the mass of the spacecraft at launch. The asteroids to be visited are automatically selected out of a large database of possible candidates. The initial shortlist of targets is based on the Minimum Orbit Intersection Distance (MOID) between the orbit of the asteroids in the database and the initial orbit of the spacecraft traversing the main belt. The final sequence is then obtained with an efficient deterministic branch and prune algorithm. The transfers between asteroids are designed using a low-thrust analytical model that provides a good estimation of the propellant consumption and transfer time. The mission analysis is completed with a study of the cost of the launch. In this paper two databases will be analysed: one containing only targets with a particular scientific relevance and one containing all available asteroids.
\end{abstract}

\footnotetext{
*Corresponding author

Email addresses: marilena.di-carlo@strath.ac.uk (Marilena Di Carlo), massimiliano.vasile@strath.ac.uk (Massimiliano Vasile), jamie.dunlop.2013@uni.strath.ac.uk (Jamie Dunlop)
} 
Keywords: main asteroid belt, low-thrust mission

\section{Introduction}

The main belt houses the majority of the asteroids in the Solar System. It extends from 2.1 AU to $4 \mathrm{AU}$ (Minton (2009) and is estimated to contain several million asteroids, ranging in size from few millimeters to the $959 \mathrm{~km}$ diameter of Ceres (Millis et al. (1987)). Although larger asteroid are observable from Earth and are easy to identify, the classification of smaller objects still remains an open problem. Furthermore, there is an interest in a characterisation of the larger ones to better understand their composition and evolution from the primordial stages of the Solar System till now. Key information on the composition of objects in the main belt can only be obtained from space-based spectroscopy and close encounter analysis (Bowles et al. (2017)). A mission that could visit at least ten objects will double the number of asteroids visited to date. However, designing a mission to characterise that many asteroids in the main belt is not an easy task. The main difficulty is to identify long sequences of asteroids that can be visited in a given time and with limited $\Delta V$. The number of known objects exceeds $641,933^{1}$ and the number of possible combinations of encounter is unmanageable.

The mission currently targeting objects in the main belt, Dawn ${ }^{2}$, is visiting only two proto-planets using low-thrust propulsion. After visiting Vesta in 2011-2013 (Rayman \& Mase (2014)), Dawn is now exploring the dwarf planet Ceres (Russell et al. (2016)).

Examples of previous works on the design of asteroid tours divided the design process into different steps (Olympio (2011)): the first step consists in the definition of a shortlist of potential targets, based on their orbital elements, dimensions or scientific characteristics. In the second step a sequence of target objects is selected using some form of global optimisation (Alemany \& Braun (2011)) in combination with reduced models that provide a quick estimation of the cost of the transfer. The last step is the optimisation of the sequence with a local optimisation method. A recent work by Sanchez Cuartielles et al. (2016) proposes a mission to fly-by 10 or more asteroids in 7 years in the timeframe 2029-2030, using a spacecraft equipped with a

\footnotetext{
${ }^{1}$ http://ssd.jpl.nasa.gov/sbdb_query.cgi\#x

${ }^{2}$ http://dawn.jpl.nasa.gov/
} 
chemical engine launched by a Soyuz launcher. The study includes the possible use of a gravity assist of Mars. A branch and bound search method is used to identify an optimal sequence of asteroids under the assumption that all fly-by's with the asteroids occur at the point of Minimum Orbital Intersection Distance (MOID). The use of gravity assist to reach the main belt is studied also by Chen et al. (2014), who present the results of an analysis of accessibility for 200 asteroids in the main belt with diameter greater than 100 $\mathrm{km}$. The global minimum of the cost of the transfer to these asteroids, using gravity assists with Mars or Earth, is sought. Results show that Mars is the most useful gravity-assist body and that dual gravity assists with Mars are the best type of trajectories to reach mid or outer belt asteroids and highinclination ones. The low-thrust transfer to asteroid Flora is included in the analysis. The control profile for the low-thrust transfer is optimised with an indirect optimal control method and homotopy. Shang \& Liu (2017) proposed a machine learning method based on Gaussian Process Regressions to study the accessibility of more than 600,000 main belt asteroids considering rendezvous realised through bi-impulsive or Mars gravity-assisted transfers. Mars' gravity assists to reach the main belt are also proposed by Yang et al. (2015). In this work, the transfer from a near-Earth asteroid to a main belt asteroid, using low-thrust propulsion and multiple gravity assists, is studied. Based on an analysis of the Tisserand graph, the Earth-Mars-Mars gravity assists sequence is found to be the best option to reach the main belt. A global solver is then used to obtain the event dates for the gravity assists and the deep space manoeuvres, using an impulsive model for the transfers. Finally, the optimal control problem for the design of the low-thrust trajectory is solved using an indirect method and homotopy.

In recent times, the problem of visiting multiple asteroids was part of the objective of some Global Trajectory Optimisation Competitions (GTOC) ${ }^{3}$. In particular, in GTOC4 the problem was to identify the maximum number of asteroids' fly-bys from a given list of 1438 objects, considering a rendezvous with the last asteroid in the sequence and a total mission time of ten years. GTOC5 also proposed a mission to Near Eearth Asteroids (NEAs) (Bottke (2000); Stuart (2001)), considering a database of 7073 objects, while GTOC7 presented a multi-spacecraft exploration of the asteroid belt and a database of 16256 potential targets. For GTOC4, the first ranking team found a solution

\footnotetext{
${ }^{3}$ https://sophia.estec.esa.int/gtoc_portal/
} 
visiting 44 asteroids (Grigoriev \& Zapletin (2013)). They solved the discrete part of the problem (the identification of the optimal sequence of asteroids to visit) using dynamic programming, performing the construction of the solution vector step by step and optimising time and mass consumption at each step. The trajectory was approximated with a solution of the Lambert problem. Lantoine \& Russell (2012) used an algorithm called HDDP (Hybrid Differential Dynamic Programming), a variant of the classical Differential Dynamic Programming technique. The multi-phase formulation of HDDP was used by splitting the trajectory into several portions connected by the fly-by's at the asteroids. The initial guess was obtained from a ballistic Lambert solution that provided the asteroid sequence. The solution was characterised by 24 fly-by's and 1 rendezvous.

This paper presents some results for a possible tour of the main asteroid belt using solar electric propulsion. The particular problem presented in this paper is similar to the one proposed in Sanchez Cuartielles et al. (2016) and differs from previous analyses of the accessibility of the main belt or asteroid tours considered in past GTOCs. More specifically, in order to limit mission time and total mass at launch, the strategy proposed in this paper is to fly-by as many asteroids as possible at their nodal points by traversing the asteroid belt with an elliptical orbit with perihelion at (or near) the Earth and aphelion at the main belt. Each asteroid is expected to be visited with one single fly-by only (see Di Carlo et al. (2017b), Vroom et al. (2016)). The resulting combinatorial problem is solved with a combination of two simple pruning techniques over the space of possible fly-by's. The first pruning is on the MOID between the initial orbit and the asteroids in the database. After this first pruning a deterministic branch and prune algorithm is applied to a binary tree that incrementally constructs the optimal sequence of targets. Finally, the best solution is re-optimised with electric propulsion. A direct transcription method based on asymptotic analytical solutions to the accelerated Keplerian motion (Zuiani et al. (2012)) is used to transcribe the optimal control problem that defines the optimal control profile of the engine. The transfer from the Earth to the initial elliptical orbit traversing the main belt is then optimised with the same transcription approach.

The length of the tour is constrained by a given total mission time and desirable launch capability. Two scenarii are considered: in the first scenario the database of target objects includes scientifically interesting bodies and tries to find the longest sequence of objects in a given time and $\Delta V$ budgets; in the second scenario, more than 100,000 objects are added to the previous 
database and the aim is find the longest sequence of asteroids that contains also some (more than 0) scientifically interesting targets. Note that the number of possible targets is, in this case, about one order of magnitude larger than the one of previous GTOCs. The analysis proceeds, as in the first scenario, with the study of all optimal sequences that are achievable with a given time limit and $\Delta V$ budget.

In this paper we limit our attention to transfer options that do not include swing-by's of the inner planets of the solar system; this reduces the complexity of the mission and improve the flexibility of the launch window. The transfer from the Earth to the main belt is, instead, conceived to exploit at best the use of the launcher and the electric propulsion system. The launch feasibility, using two possible launchers, is also studied.

The paper is structured as follows: the proposed solution method to define a mission to the main belt is presented in Section 2. The results are then presented in Sections 3 and 4 and Section 5 concludes the paper.

\section{Mission analysis}

In this work two databases of objects in the main belt are considered. The first database (Database 1) includes a selection of 424 objects of particular scientific interest (the database was kindly provided by the PI of the CASTAway mission proposal ${ }^{4}$ ). These are, among others, active objects (main belt comets, mass losing asteroids), objects of extreme sizes (both small and big) and extreme shapes, fast rotators, binaries or triples and asteroid pairs. The second database (Database 2) is composed of 101,993 objects (Sanchez Cuartielles et al. (2016)). The distribution of semimajor axis $a$, eccentricity $e$ and inclination $i$ of the objects of the two databases is shown in Figures 1 and 2 , where the curve $q=Q_{\text {Mars }}$ identifies the values of $a$ and $e$ such that the perihelion $q$ is equal to the aphelion of Mars $Q_{\text {Mars }}$ while $Q=q_{\text {Jup }}$ identifies the values of $a$ and $e$ such that the aphelion $Q$ is equal to the perihelion of Jupiter. Note that, although the complete Database 2 contains also asteroids with perihelion at Jupiter, in this analysis we will restrict our attention to asteroids that are part of the main belt.

The approach taken in this paper to design the tour of the asteroid belt is conceptually analogous to the one proposed by the authors in previous work

\footnotetext{
${ }^{4}$ https://sites.google.com/site/castawaymission/
} 

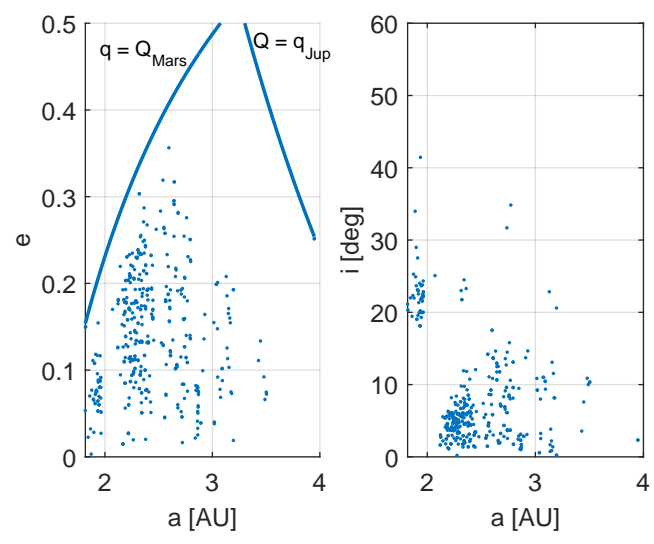

Figure 1: $a-e$ and $a-i$ distribution of the selected objects in the main belt for Database 1.

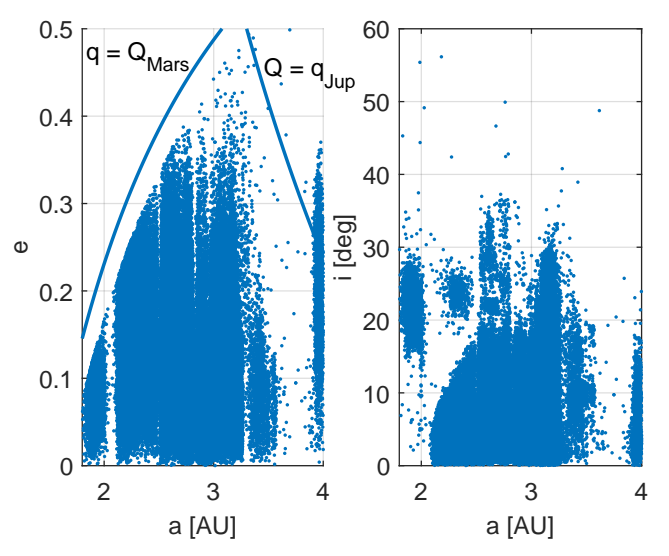

Figure 2: $a-e$ and $a-i$ distribution of the selected objects in the main belt for Database 2 .

(see Di Carlo et al. (2017b)) and formulates the underlying trajectory design problem in the same fashion as in Sanchez Cuartielles et al. (2016). The idea is to traverse the asteroid belt with a high elliptical orbit and to encounter as many asteroids as possible at their nodal points to avoid any plane change. Thus the orbit of the spacecraft is designed to be in the ecliptic plane and electric propulsion is used to modify the elliptical orbit enough to obtain the required fly-by distance. Different planes could potentially increase the length of the sequence of encounters though it would incur in an additional $\Delta V$. The use of one or more swing-by's of the inner planets could mitigate this problem and increase both aphelion and perihelion but also increase the operational complexity of the mission. Therefore, the analysis in this paper will be limited to the case in which no swing-by's are introduced and the orbit of the spacecraft remains in the ecliptic plane.

The tour of the main belt is assumed to start on the 01/01/2030 with a maximum duration of 5 years to limit operations and respond to the requirements and scientific objectives of CASTAway. The spacecraft is injected in a heliocentric orbit exploiting the $C_{3}$ provided by the launcher and then uses the electric engine to achieve the desired elliptical orbit. Note that other dates around the 01/01/2030 were analysed but it was found that the 01/01/2030 was optimal, thus this paper will present only the results for the 01/01/2030 as they are the most significant ones (Section 4.2).

The overall approach can be conceptually divided in five steps that are briefly introduced in the following and described in more details in the next 
subsections:

1. Analysis of the MOID between different possible initial orbits of the spacecraft traversing the main belt and the orbits of all the asteroids in the database (Subsection 2.1). This is a natural step to shortlist groups of asteroids that can be easily reached with a fly-by within the desired time frame. Note that the idea of using the MOID descends from the idea of traversing the main belt with a high elliptical orbit.

2. Definition of the sequence of asteroid close encounters starting from the shortlist derived from step 1. The MOIDs in the shortlist might be excessively big to allow achieving the scientific goals of the mission, therefore, the elliptical orbit needs to be modified to guarantee an appropriate distance from each asteroid. Such a modification incurs in an additional $\Delta V$ cost that needs to be traded-off against the number of close encounters (Subsection 2.2). For this step we use a multi-impulse model and a simple but effective binary decision tree coupled with a deterministic branch and prune approach.

3. Optimisation of the parameters of the initial orbit in the main belt, of the times of the impulsive maneuvers and of the times of the encounters with the asteroids to reduce the $\Delta V$ associated with the tour (Subsection 2.3). This step, although not strictly required, provides an optimised impulsive solution that can be used as it is or recalculated considering an electric propulsion engine.

4. Study of the transfer from the Earth to the main belt (Subsection 2.4).

5. Optimisation of the low-thrust transfer to the main belt and of the tour of the selected sequence of asteroids (Subsection 2.5). For this step we used a novel transcription method that was already demonstrated to be fast and effective and to provide conservative but good estimations of the total mass budget.

The analysis of the transfer to the main belt and of the encounter sequence is then completed by a launch sequence analysis to assess which launch system can be used for this particular mission.

\subsection{Minimum Orbit Intersection Distance}

In order to identify the initial orbit of the spacecraft and shortlist the asteroids to encounter, the MOID (Gronchi $(2002,2005)$ ) between all the asteroids in the database and different possible initial orbits of the spacecraft is computed. The MOID is defined as a measure for the distance between 
the orbits of two objects. The computation of the MOID is realised using the Fortran code publicly available online from the Department of Mathematics of the University of Pisa, Italy ${ }^{5}$. This computation returns, for each pair spacecraft's orbit-asteroids's orbit, the minimum, maximum and saddle points of the distance between the two orbits. These critical points are identified by the true anomalies $\theta_{a s t}^{M O I D}$ and $\theta_{s c}^{M O I D}$ of the two objects on their orbit and by the distance between them at the critical points, $d$. In this study only points with $d<0.01 \mathrm{AU}$ are considered. The computation of the MOID does not consider, however, the positions that the asteroids and spacecraft occupy on their orbits (Bonanno (2000)). This means that an encounter between spacecraft and asteroid can not actually take place if the two bodies are not, at the same time, at $\theta_{a s t}^{M O I D}$ and $\theta_{s c}^{M O I D}$. In order to check which encounters at the MOID can be actually be realised, the following phasing analysis is applied:

- For each couple spacecraft's orbit - asteroid's orbit with $d<0.01$ AU, the times when the asteroid is at $\theta_{\text {ast }}^{\text {MOID }}$ are computed, starting from the initial date $01 / 01 / 2030, t_{0}=10957.5$ MJD2000. These times, that repeat at intervals equal to the orbital period of the asteroid, are identified as $T_{\text {ast }}^{M O I D}$.

- Different initial mean anomalies $M_{0}$ in the range $[0,360)$ deg, at steps of $1 \mathrm{deg}$, are considered for the spacecraft on its orbit, with initial date $t_{0}$.

- Kepler equation is solved to obtain the true anomaly of the spacecraft at $T_{s c}^{M O I D}, \theta_{s c}\left(T_{a s t}^{M O I D}\right)$, starting from $M_{0}$ at $t_{0}$. If the following condition is satisfied

$$
\left|\theta_{s c}\left(T_{a s t}^{M O I D}\right)-\theta_{s c}^{M O I D}\right|<\delta
$$

then the encounter between asteroid and spacecraft, at distance $d<0.01$ AU, actually takes place at time $T_{a s t}^{M O I D} . \delta$ is an appropriate small angle.

\subsection{Study of the possible sequences of asteroids}

At the end of the process defined in the previous subsection, for each value of $M_{0}$ (angular position of the spacecraft on the initial orbit at time

\footnotetext{
${ }^{5}$ http://adams.dm.unipi.it/ gronchi/HOMEPAGE/research.html
} 
$t_{0}$ ), a list of asteroids that the spacecraft encounters at a distance lower than $0.01 \mathrm{AU}$ is available. The next step consists in computing the $\Delta V$ required to fly-by these objects. The cost of the transfer between one asteroid and the next is computed with a Lambert solver (Vallado (2007)). The total cost is given by the sum of all the $\Delta V_{i}, \Delta V=\sum_{i} \Delta V$. Encountering each asteroid in the sequence could be however too expensive in terms of $\Delta V$. This study, therefore, tries to identify a subset of objects, in the list of asteroids, that can be visited with a cost lower than a maximum allowable total $\Delta V_{\max }$ budget. In order to do so, for a sequence of $n$ asteroids, a vector $\mathbf{b}$ of length $n$ composed of 0's and 1's is defined to identify which asteroids are encountered (1) and which ones are not $(0)$. As a result, $2^{n}$ sequences, each characterised by a different number of asteroids and different values of $\Delta V$, are available and need to be evaluated. An enumerative approach to evaluate all the $2^{n}$ possibilities is not practical when $n$ is large. Thus a deterministic Branch and Prune Approach (BPA) is applied. The BPA incrementally builds a binary tree in which each level corresponds to one of the $n$ components in $\mathbf{b}$ and each branch is a sequence. At each level each branch is divided in two sub-branches, one with leaf with value 1 and one with leaf with value 0 . Then each partial branch is evaluated. If the $\Delta V$ associated to the partial branch exceeds a given threshold the whole branch is discarded. This simple approach has guaranteed convergence to the global optimum for a given allowable $\Delta V_{\max }$ budget and is very fast. The major limitation is represented by the required memory storage if the upper limit on the $\Delta V$ budget is increased. In this case the length of the sequence also increases but the total volume of possible alternative sequences grows considerably. Future work will be devoted to have an adaptive adjustment of the threshold to allow a better exploration while limiting the excessive use of memory. A graphical representation of the binary tree and of its working mechanism is given in Figures 3 and 4.

After this process, for each value of $M_{0}$ on the initial orbit, the vector b is translated into a list of $N$ asteroids $\mathcal{A}=\left\{A_{1}, A_{2}, A_{3}, \ldots A_{N}\right\}$, with $N \leq n$ and $\Delta V \leq \Delta V_{\max }$. The initial orbit of the spacecraft is defined by means of its orbital elements: $\mathcal{O} \mathcal{E}=\left\{a, e, i, \Omega, \omega, M_{0}, t_{0}\right\}$, where $\Omega$ is the right ascension of the ascending node, $\omega$ is the argument of perihelion and $M_{0}$ is the mean anomaly at time $t_{0}$. The dates of the encounters are defined as $\mathcal{T}=\left\{T_{1}, T_{2}, \cdots T_{N}\right\}$. 


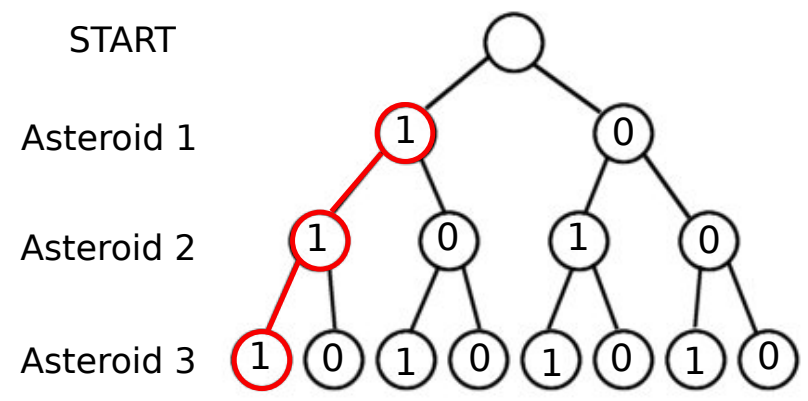

Figure 3: Representation of the binary tree. Each asteroid in the sequence can be assigned a value equal to 0 or 1 (left); only asteroids with associated value of 1 are visited (right). Branches with $\Delta V$ higher than threshold (example, in red in the figure) are discarded.
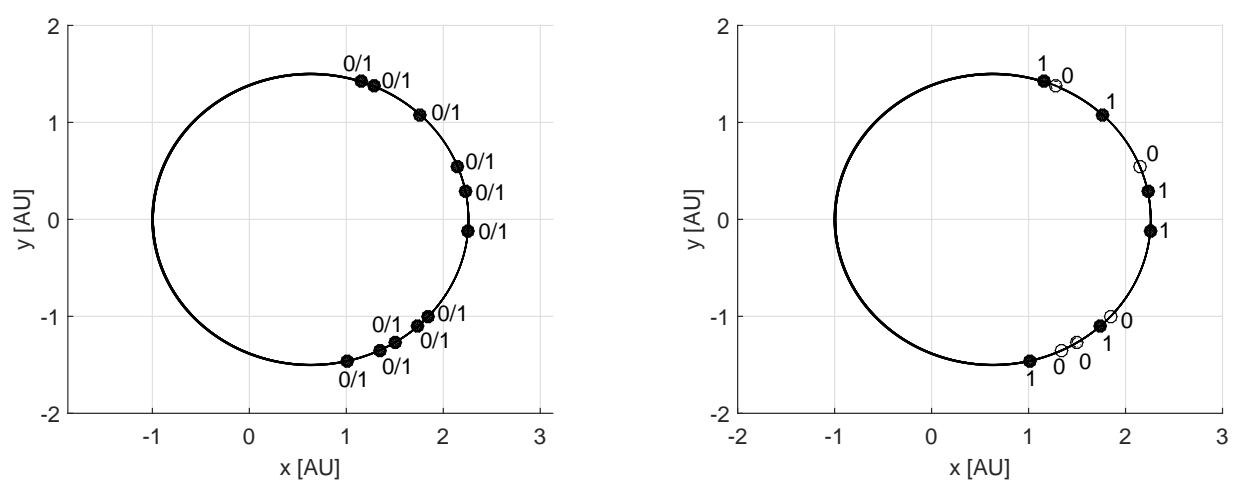

Figure 4: Identification of the sequence of asteroids to visit using the binary tree. Each asteroid in the sequence can be assigned a value equal to 0 or 1 (left); only asteroids with associated value of 1 are visited (right).

\subsection{Optimisation of the sequence of asteroids}

The solution found at the previous step assumes that the encounters with the asteroids take place when they are at their critical true anomalies, $\theta_{a s t}^{M O I D}$, starting from an initial orbit identified by $\mathcal{O E}$. A better solution might however exist and could be found by changing some of the parameters of the initial orbit $\mathcal{O E}$ (the initial mean anomaly $M_{0}$, the semimajor and eccentricity $a$ and $e$ and the argument of perigee $\omega$ ) or by changing the dates of encounters with the asteroids $\mathcal{T}$, that is by encountering the asteroids not exactly at $\theta_{\text {ast }}^{M O I D}$. In order to find a better solution, a continuous global optimisation 
problem is solved, in which the objective is the minimisation of the total $\Delta V$. The upper and lower boundaries for the global optimisation problem are defined by the vectors $\mathbf{L B}$ and $\mathbf{U B}$ :

$$
\mathbf{L B}=\left[M_{0}-\Delta M_{0}, a-\Delta a, e-\Delta e, \omega-\Delta \omega, T_{1}-\Delta T_{1}, T_{2}-\Delta T_{2}, \ldots T_{n}-\Delta T_{n}\right]^{T}
$$

$$
\mathbf{U B}=\left[M_{0}+\Delta M_{0}, a+\Delta a, e+\Delta e, \omega+\Delta \omega, T_{1}+\Delta T_{1}, T_{2}+\Delta T_{2}, \ldots T_{n}+\Delta T_{n}\right]^{T}
$$

The global search is realised using the global optimiser Multi Population Adaptive Inflationary Differential Evolution Algorithm (MP-AIDEA) (Di Carlo et al. (2015)). MP-AIDEA is a multi-population adaptive version of Inflationary Differential Evolution which combines Differential Evolution (DE) (Price et al. (2006)) with the working principles of Monotonic Basin Hopping Algorithm (MBH) (Wales \& Doye (1997)) (see Vasile et al. (2011)). MP-AIDEA has been extensively tested on various benchmarks of difficult problems and was shown to be very effective at solving complex trajectory optimisation problems compared to other state-of-the-art global optimisation methods.

\subsection{Transfer from Earth to the main belt}

This section describes the transfer strategy from the Earth to the first orbit in the main belt, $\mathcal{O E}=\left\{a, e, i, \Omega, \omega, M_{0}, t_{0}\right\}$. The transfer is realised by injecting the spacecraft into an intermediate phasing orbit, characterised by orbital elements $\mathcal{O} \mathcal{E}_{\text {int }}=\left\{a_{\text {int }}, e_{\text {int }}, i, \Omega, \omega, 0, T_{L}\right\}$ and orbital period $T_{\text {int }}$. $T_{L}$ is the date of the launch of the spacecraft and the corresponding mean anomaly is zero because, at launch, the spacecraft is at the perihelion of the orbit (Earth). The $\Delta V$ required for the launch, $\Delta V_{L}$, is computed as:

$$
\Delta V_{L}=\sqrt{2 \frac{\mu_{\odot}}{r_{\oplus}}-\frac{\mu_{\odot}}{a_{i n t}}}-\sqrt{\frac{\mu_{\odot}}{r_{\oplus}}}
$$

where $\mu_{\odot}$ is the Sun's planetary constant and $r_{\oplus}$ is the Sun-Earth distance. The spacecraft remains on the intermediate phasing orbit for an integer number $n_{\text {rev }}$ of revolutions. After $n_{\text {rev }}$ revolutions, when the spacecraft is at the perihelion $r_{\oplus}$ of the intermediate phasing orbit, $\Delta V_{M}$ is applied to reach the final orbit of semimajor axis $a$ :

$$
\Delta V_{M}=\sqrt{2 \frac{\mu_{\odot}}{r_{\oplus}}-\frac{\mu_{\odot}}{a}}-\sqrt{2 \frac{\mu_{\odot}}{r_{\oplus}}-\frac{\mu_{\odot}}{a_{i n t}}}
$$


The spacecraft moves then for a time:

$$
\Delta T=\frac{M_{0}-M_{p}}{n}
$$

on the orbit $\mathcal{O} \mathcal{E}$. In the previous equation $M_{0}$ is the mean anomaly on the first orbit in the main belt at $t_{0}, M_{p}=0 \mathrm{deg}$ is the mean anomaly at perihelion and $n$ is the mean motion of the orbit $\mathcal{O E}$. For every value of $n_{\text {rev }}$, $T_{\text {int }}$ has to be such that at the computed time of the launch, $T_{L}$ :

$$
T_{L}=t_{0}-\Delta T-n_{r e v} T_{i n t}
$$

the Earth is at the perihelion of $\mathcal{O} \mathcal{E}$. This allows one to identify the value of $T_{\text {int }}$, and, therefore, the intermediate phasing orbit $\mathcal{O} \mathcal{E}_{\text {int }}$, for every $\mathcal{O} \mathcal{E}$ and $n_{\text {rev }}$.

The method described above provides an impulsive solution for the transfer from Earth to $\mathcal{O} \mathcal{E}$ based on the assumption that the spacecraft is injected by the launcher or an upper stage into $\mathcal{O} \mathcal{E}_{\text {int }}$. When the actual launch capabilities are considered the $\Delta V$ required to inject the spacecraft into the orbit $\mathcal{O} \mathcal{E}_{\text {int }}$, by means of an escape hyperbola characterised by $V_{\infty}=\Delta V_{L}$, is the sum of two contributions:

$$
\Delta V_{\text {total }}=\Delta V_{i}\left(i_{i n j}, \omega_{i n j}\right)+\Delta V_{i n j}\left(i_{i n j}, \omega_{i n j}\right)
$$

where $\Delta V_{i}\left(i_{i n j}, \omega_{i n j}\right)$ and $\Delta V_{i n j}\left(i_{i n j}, \omega_{i n j}\right)$ are, respectively, the $\Delta V$ required to change inclination from the orbit where the launcher is injecting the spacecraft to the escape hyperbola and the $\Delta V$ required to reach the required escape velocity. These $\Delta V^{\prime}$ 's are a function of $i_{i n j}$ and $\omega_{i n j}$, the inclination and argument of the pericentre of the escape hyperbola. The calculation of these two $\Delta V$ 's follows the approach presented in Di Carlo et al. (2017b). No consideration is done about the right ascension of the asymptote because any required right ascension of departure may be achieved by changing the time of day at which the spacecraft is launched (Kemble (2006)). Once $\Delta V_{\text {total }}$ has been found, the dry and propellant masses of the upper stage, $m_{d r y}^{U / S}$ and $m_{\text {prop }}^{U / S}$, can be found from: 


$$
\begin{aligned}
& \frac{m_{\text {prop }}^{U / S}}{m_{\text {prop }}^{U / S}+m_{d r y}^{U / S}}=k \\
& m_{0}+m_{d r y}^{U / S}=\left(m_{d r y}^{U / S}+m_{\text {prop }}^{U / S}+m_{0}\right) \exp \left(-\frac{\Delta V_{\text {total }}}{I_{s p}^{U / S} g_{0}}\right)
\end{aligned}
$$

where $k$ and $I_{s p}^{U / S}$ are the propellant mass fraction and specific impulse of the upper stage and $m_{0}$ is the initial wet mass of the low-thrust spacecraft. In this paper it is assumed that the launcher injects the spacecraft in a Geostationay-Transfer Orbit (GTO). The total payload mass that the launcher has to inject into GTO is, therefore, $m_{p l}=m_{0}+m_{\text {prop }}^{U / S}+m_{d r y}^{U / S}$.

\subsection{Low-thrust optimisation}

The outcome of the sequence finder and optimisation with MP-AIDEA is a sequence of transfer legs characterised by a departure heliocentric position, an end heliocentric position, a transfer time and a departure $\Delta V$. The lowthrust optimisation process determines, for each transfer leg, an optimal control history, for the low-thrust engine, to depart from one asteroid and reach the following asteroid in the sequence at a given time. The same process is applied also to optimise the transfer from Earth to $\mathcal{O E}$. In this study, a variant of the direct analytical multiple shooting algorithm proposed by Zuiani et al. (2012) and implemented in the software code FABLE (FAst Boundaryvalue Low-thrust Estimator) is used. More information about FABLE can be found in Di Carlo et al. (2017a). The transfer leg is split into a predefined sequence of $n_{L T}$ finite coast and thrust arcs. Each $s$-th arc is represented by a vector of equinoctial parameters $\mathbf{E}_{s}=\left[a_{s}, P_{1, s}, P_{2, s}, Q_{1, s}, Q_{2, s}, L_{s}\right]^{T}$, plus, in case of thrust arc, the low-thrust acceleration components, $a_{r}, a_{t}$ and $a_{h}$ expressed in a local radial-transversal reference frame as (Zuiani et al. (2012)):

$$
\mathbf{a}_{L T, s}=\left\{\begin{array}{l}
a_{r} \\
a_{t} \\
a_{h}
\end{array}\right\}_{s}=\left\{\begin{array}{c}
\epsilon_{s} \cos \alpha_{s} \cos \beta_{s} \\
\epsilon_{s} \sin \alpha_{s} \cos \beta_{s} \\
\epsilon_{s} \sin \beta_{s}
\end{array}\right\}
$$

where $\alpha_{s}, \beta_{s}$ and $\epsilon_{s}$ are, respectively, the azimuth, elevation and modulus of the acceleration and $\epsilon_{s}=F / m_{s}$ is the ratio between the thrust $F$ and the current mass of the spacecraft $m_{s}$. The mass of the spacecraft is conservatively kept constant over each transfer arc and updated at the end of the 


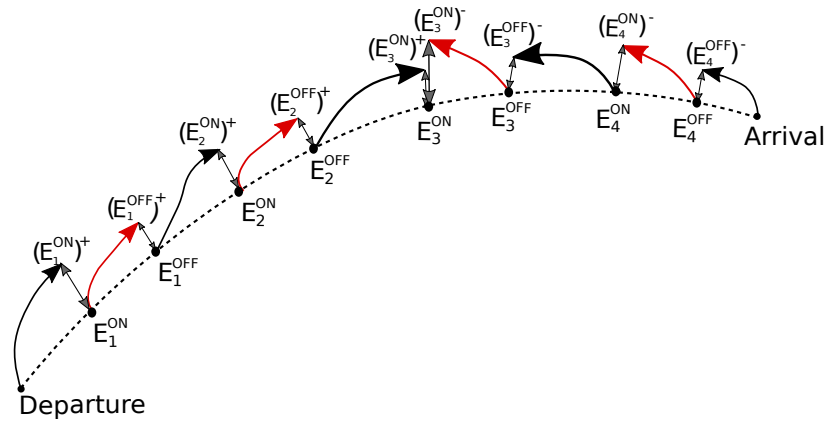

Figure 5: Segmentation of the trajectory into coast arcs (black) and thrust arcs (red). Each node is identified by a vector of equinoctial parameters, $\mathbf{E}_{s}$. In particular, $\mathbf{E}^{O N}$ nodes define the switching point from a coast to a thrust arc while $\mathbf{E}^{O F F}$ nodes define the switching point from a thrust to a coast arc.

transfer according to the propellant mass spent to realise that transfer. In the following the initial mass of the spacecraft at launch $m_{0}$ will be set to a predefined value of $1000 \mathrm{~kg}$. Note, however, that this assumption does not limit the validity of the results as any other initial mass at launch $m_{0}^{\prime}$ can be used, provided that the thrust magnitude is scaled by the ratio $m_{0}^{\prime} / m_{0}$. The trajectory is analytically propagated forward from the departure point and backward from the end point (Figure 5). The motion is assumed purely Keplerian along coast arcs while thrust arcs are analytical propagated using the asymptotic expansion solutions proposed in the work of Zuiani \& Vasile (2015). Each arc begins and ends at an On/Off control node, where On nodes define the switching point from a coast to a thrust arc and Off nodes define the switching point from a thrust to a coast arc (see Figure 5). Therefore, thrust arcs are defined by a set of orbital elements at an On node, $E_{s}^{O N}$, and coast arcs are defined by a set of orbital elements at an Off node, $E_{s}^{O F F}$. The approach implemented in FABLE was shown to provide fast convergence to nearly optimal solutions. For more information the interested reader can refer to Zuiani et al. (2012) and Zuiani \& Vasile (2015). For the trajectories considered in this study, the angle $\beta$ is set to zero, since the transfers are all on the ecliptic plane and require no change of inclination (Section 3 and 4). This is a consequence of the assumptions and methods described in Section 2: the fly-bys of the asteroids take place at the MOID points of the spacecraft's heliocentric elliptic orbit and asteroids' orbits. The azimuth angles $\alpha_{s}$ are, instead, optimisation variables. The optimisable vector for each transfer 
is, therefore, defined by the azimuth angles $\alpha_{s}$, for each thrust arc, and the equinoctial elements at each On and Off point:

$$
\mathbf{x}_{L T}=\left[\alpha_{1}, \mathbf{E}_{1}^{O N}, \mathbf{E}_{1}^{O F F}, \alpha_{2}, \mathbf{E}_{2}^{O N}, \mathbf{E}_{2}^{O F F}, \ldots, \alpha_{n_{L T}}, \mathbf{E}_{n_{L T}}^{O N}, \mathbf{E}_{n_{L T}}^{O F F}\right]^{T}
$$

The optimisation problem is formulated as the following non-linear programming problem whose objective is the total $\Delta V$ for each transfer:

$$
\min _{\mathbf{x}_{L T}} \Delta V=\sum_{s} \epsilon_{s} \Delta t_{s}\left(\mathbf{x}_{L T}\right)
$$

where $\Delta t_{s}\left(\mathbf{x}_{L T}\right)$ is the time length of each thrust arc, subject to the following constraints:

$$
\begin{cases}\left(\mathbf{E}_{1}^{O N}\right)^{+}=\mathbf{E}_{1}^{O N} & \\ \left(\mathbf{E}_{s}^{O F F}\right)^{+}=\mathbf{E}_{s}^{O F F} & s=1, \ldots, n_{L T} / 2 \\ \left(\mathbf{E}_{s}^{O N}\right)^{-}=\mathbf{E}_{s}^{O N} & s=n_{L T} / 2+1, \ldots, n_{L T} \\ \left(\mathbf{E}_{n L T / 2+1}^{O N}\right)^{+}=\left(\mathbf{E}_{n L T / 2+1}^{O N}\right)^{-} & \\ \left(\mathbf{E}_{n L T}^{O F F}\right)^{-}=\mathbf{E}_{n L T}^{O F F} & \\ \sum_{s=1}^{n_{L T}} \Delta t_{s}=T o F & \end{cases}
$$

The plus and minus signs in the constraints equations indicate, respectively, the forward integration arc and the backward integration arc. The non-linear programming problem is solved using the Matlab ${ }^{\circledR}$ fmincon-interior-point algorithm.

\section{Results Database 1}

The first search for optimal tours considers the asteroids in the database of scientific interesting asteroids (Database 1). This section presents the results of the scan of all possible sequences, with estimated cost lower than $\Delta V_{\max }=1 \mathrm{~km} / \mathrm{s}$, and the low-thrust optimisation of the most promising solution.

\subsection{Minimum Orbit Intersection Distance}

The MOID is computed between all the asteroids in the database and different orbits of the spacecraft identified by the orbital elements in Table 1. The spacecraft orbits are elliptical, with perihelion $r_{p}$ at the Earth and 
Table 1: Orbital elements of the different possible initial orbits of the spacecraft used for the computation of the MOID with the asteroids of Database 1.

\begin{tabular}{lllll}
\hline$r_{p}[\mathrm{AU}]$ & $r_{a}[\mathrm{AU}]$ & $i[\mathrm{deg}]$ & $\Omega[\mathrm{deg}]$ & $\omega[\mathrm{deg}]$ \\
\hline 1 & {$[1.8,4]$} & {$[0,30]$} & 0 & {$[0,360]$} \\
\hline
\end{tabular}

aphelion $r_{a}$ in a given range of distances from the Sun. Figure 6 shows, for the considered values of $r_{a}, \omega$ and $i$, the number of asteroids with $d<$ $0.01 \mathrm{AU}$ with respect to the orbit of the spacecraft. Results show that this number increases with decreasing inclination. As the inclination increases a dependence on the argument of the perihelion of the orbit become also evident and large regions where the number of asteroids with $d<0.01 \mathrm{AU}$ is zero appear. 

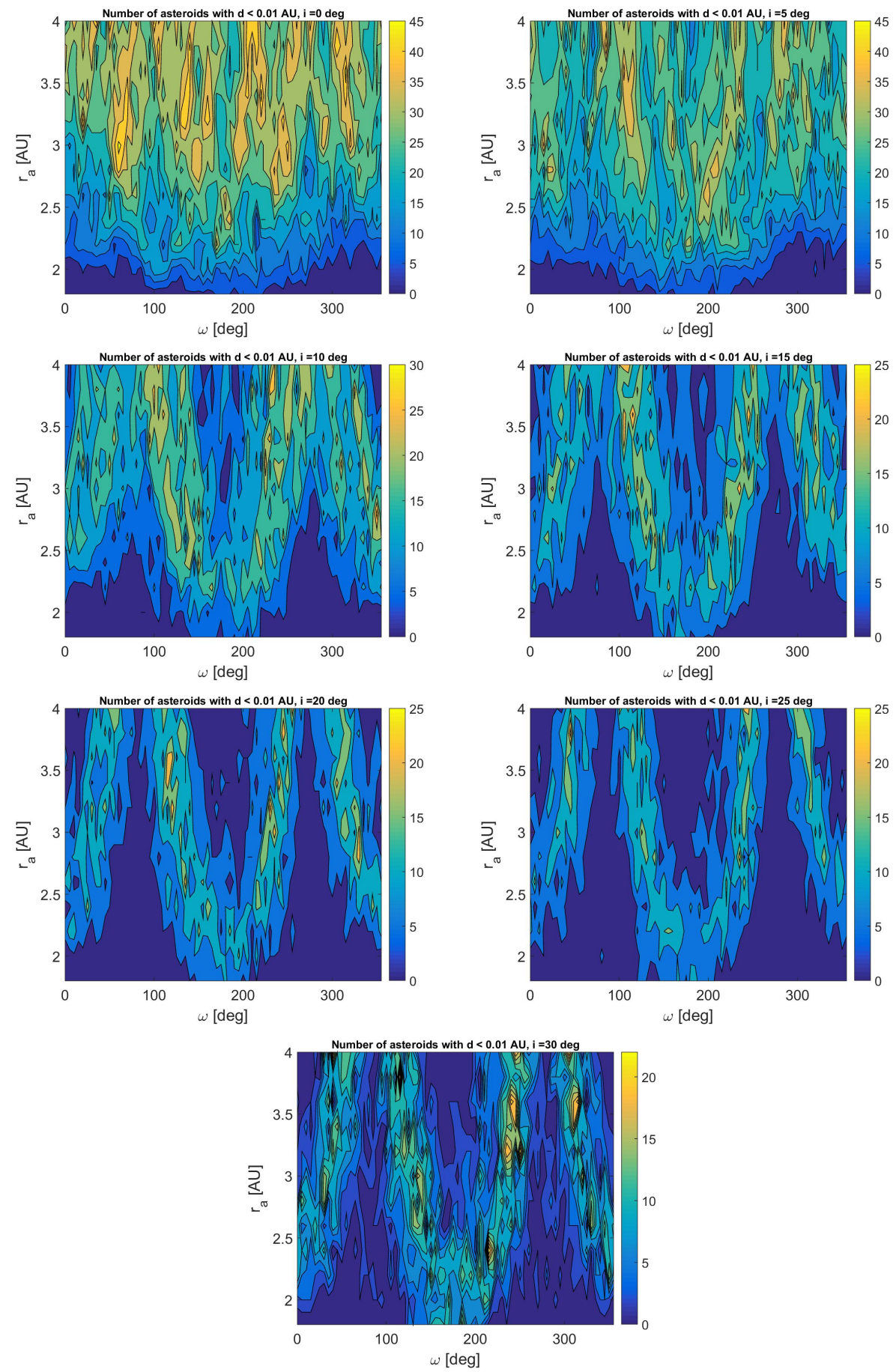

Figure 6: Number of asteroids in Database 1 with $d<0.01$ AU for different initial orbits of the spacecraft. The initial orbits of the spacecraft are identified by different values of $r_{a}$ ( $y$ axis of each figure), $\omega$ ( $x$ axis) and $i$, from $i=0 \operatorname{deg}$ (top left) to $i=30 \operatorname{deg}$ (bottom). 
The number of asteroids shown in Figure 6 does not account for the position of asteroids and spacecraft on their orbits. Once the phasing process presented in Subsection 2.1 is applied, the number of possible asteroids to encounter with $d<0.01$ is further reduced. In particular, after phasing, two orbits characterised by the highest number of encounters with the asteroids in Database 1 can be identified. The orbital elements of these two orbits (O1 and O2) are given in Table 2.

Table 2: Orbits providing the higher number of encounters with asteroids in Database 1.

\begin{tabular}{llllll}
\hline & $a[\mathrm{AU}]$ & $e$ & $i[\mathrm{deg}]$ & $\Omega[\mathrm{deg}]$ & $\omega[\mathrm{deg}]$ \\
\hline O1 & 2.2 & 0.5455 & 0 & 0 & 220 \\
O2 & 2.3 & 0.5652 & 0 & 0 & 315 \\
\hline
\end{tabular}

The number of possible encounters for different values of $M_{0}$ from 0 to $359 \mathrm{deg}$, for the orbits defined in Table 2, is shown in Figure 7. Results show that the maximum number of asteroids that it is possible to visit in 5 years is 8. The cost associated to the mission has however to be computed to verify that it is below the limit value of $\Delta V_{\max }=1 \mathrm{~km} / \mathrm{s}$.

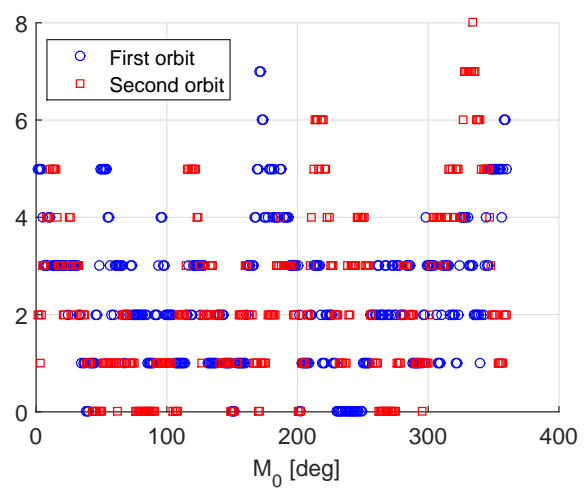

Figure 7: Number of asteroids with $d<0.01$ and phasing condition (Equation 1) satisfied.

\subsection{Study of the possible sequence of asteroids}

Figures 8 and 9 show the $\Delta V$ required for the tour of the asteroids in Database 1 , as a function of the number $N$ of objects visited, for the two orbits defined in Table 2 and $\Delta V_{\max }=1 \mathrm{~km} / \mathrm{s}$. The figures collect the results 
obtained for all the possible values of $M_{0}$ from 0 to $359 \mathrm{deg}$, at steps of 1 deg. Results show that, within the limit of $\Delta V_{\max }=1 \mathrm{~km} / \mathrm{s}$, the maximum number of asteroids that is possible to visit is $N=3$ for O1 and $N=4$ for O2. The total computation time to obtain these results, for all the values of $M_{0}$ ranging from 1 to $359 \mathrm{deg}$, is 1 second on a Intel(R) Core(TM) i7-3770 CPU $3.4 \mathrm{GHz}$ and $8 \mathrm{~GB}$ RAM using Matlab R2015a. The length $n$ of the binary vector $\mathbf{b}$ ranges from 1 to 7 , depending on $M_{0}$.

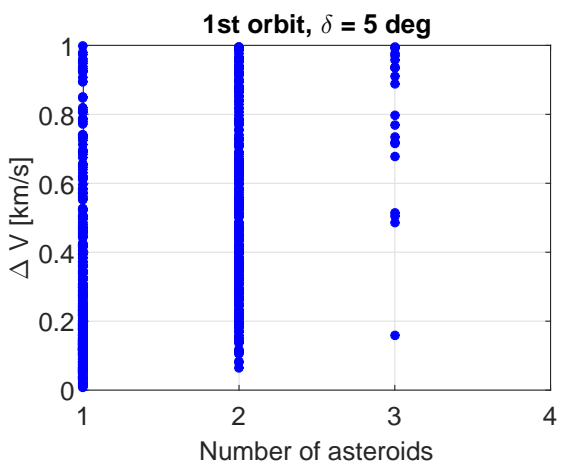

Figure 8: Relation between $\Delta V$ and number of visited asteroids for the orbit O1 for $\Delta V_{\max }=1 \mathrm{~km} / \mathrm{s}$.

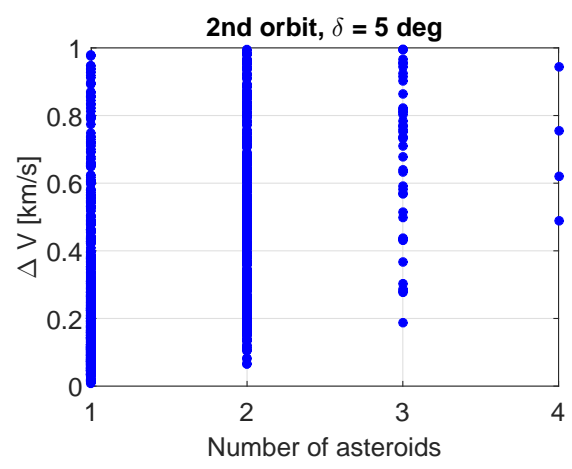

Figure 9: Relation between $\Delta V$ and number of visited asteroids for the orbit O2 for $\Delta V_{\max }=1 \mathrm{~km} / \mathrm{s}$.

Results in Figures 8 and 9 are obtained using $\delta=5 \mathrm{deg}$. The sensitivity of the results on the value of $\delta$ is presented in Table 3, where the total number of solutions $N_{T O T}$ and the number of solutions with 3 or 4 visited asteroids, $N_{3}$ and $N_{4}$, are presented for different values of $\delta$. Results show that the total number of solutions $N_{T O T}$ and the number of solutions with 3 or 4 visited asteroids decrease with $\delta$.

Table 3: Sensitivity of the number of solutions to $\delta$.

\begin{tabular}{l|ll|ll}
\hline & \multicolumn{2}{|c|}{ O1 } & \multicolumn{2}{c}{ O2 } \\
\hline$\delta[\mathrm{deg}]$ & $N_{\text {TOT }}$ & $N_{3}$ & $N_{\text {TOT }}$ & $N_{4}$ \\
\hline 5 & 1058 & 20 & 1532 & 4 \\
1 & 528 & 4 & 700 & 0 \\
0.5 & 324 & 2 & 428 & 0 \\
0.25 & 172 & 1 & 198 & 0 \\
\hline
\end{tabular}




\subsection{Optimisation of the sequence of asteroids}

The best solutions in Figures 8 and 9 are optimised with MP-AIDEA. The lower and upper boundaries LB and UB used for the optimisation with MPAIDEA are defined by Equations 2 and 3 and the values reported in Table 4. The intervals $\Delta a, \Delta e$ and $\Delta \omega$ are given as a function of the nominal values, $a, e$ and $\omega$. MP-AIDEA is run for 50,000 function evaluations and the optimisation is repeated 25 times. The best solution obtained at the end of this process is then considered.

Table 4: Parameters for the definition of UB and LB.

\begin{tabular}{lllll}
\hline$\Delta M_{0}[\mathrm{deg}]$ & $\Delta a$ & $\Delta e$ & $\Delta \omega$ & $\Delta T_{i}$ [days $]$ \\
\hline 1 & $0.01 a$ & $0.01 e$ & $0.01 \omega$ & 10 \\
\hline
\end{tabular}

Table 5 shows the best optimised $\Delta V_{\text {opt }}$, together with the number of visited asteroids $N$, the angle $\delta$ and the initial $\Delta V$ before the optimisation with MP-AIDEA.

Table 5: Optimisation of the $\Delta V$ of the longest sequence of asteroids for the two orbits defined in Table 2.

\begin{tabular}{lllll}
\hline Orbit & $N$ & $\delta[\mathrm{deg}]$ & $\Delta V[\mathrm{~km} / \mathrm{s}]$ & $\Delta V_{\text {opt }}[\mathrm{km} / \mathrm{s}]$ \\
\hline O1 & 3 & 5 & 0.1580 & 0.1024 \\
O2 & 4 & 5 & 0.4881 & 0.3057 \\
\hline
\end{tabular}

The solution selected for the low-thrust optimisation is the one associated to orbit $2(\mathrm{O} 2)$ in Table 2, as it allows to encounters 4 rather than 3 asteroids of Database 1. Details of the transfer are given in Table 6 and in Figure 10. The initial orbit in the main belt is characterised by orbital elements: $\mathcal{O E}_{1}=$ $\left\{a=2.2945 \mathrm{AU}, e=0.5652, i=0 \mathrm{deg}, \Omega=0 \mathrm{deg}, \omega=315.2038 \mathrm{deg}, M_{0}=\right.$ $\left.214.8032 \mathrm{deg}, t_{0}=10958.5 \mathrm{MJD} 2000\right\}$ Asteroid $2006 \mathrm{UJ} 47$ is a fast rotator, characterised by a rotation period of $0.64 \mathrm{~h}$. The other three asteroids in Table 6 are asteroids pairs. Asteroids pairs are defined as asteroids that had a very small relative velocity at some point in the past, in the order of $\mathrm{m} / \mathrm{s}^{6}$. They may represent former binary asteroids or the result of collisional

\footnotetext{
${ }^{6}$ http://www . johnstonsarchive.net/astro/asteroidpairs.html
} 
breakup of a parent asteroid.

Table 6: Selected solution for the main belt tour for Database 1.

\begin{tabular}{lllllll}
\hline $\begin{array}{l}\text { Targeted } \\
\text { Asteroid }\end{array}$ & \multirow{2}{*}{ Dep. Date } & $\begin{array}{l}\text { Optimised } \\
\text { Dep. Date }\end{array}$ & $\begin{array}{l}\text { ToF } \\
{[\text { days }]}\end{array}$ & $\begin{array}{l}\text { Opt. ToF } \\
{[\text { days }]}\end{array}$ & $\begin{array}{l}\Delta V \\
{[\mathrm{~m} / \mathrm{s}]}\end{array}$ & $\begin{array}{l}\text { Opt. } \Delta V \\
{[\mathrm{~m} / \mathrm{s}]}\end{array}$ \\
\hline 2006 UJ47 & $01 / 01 / 2030$ & $01 / 01 / 2030$ & 294.25 & 294.02 & 80.34 & 67.58 \\
2007 UV & $22 / 10 / 2030$ & $22 / 10 / 2030$ & 363.64 & 364.22 & 147.52 & 105.63 \\
2005 YN176 & $20 / 10 / 2031$ & $21 / 10 / 2031$ & 207.00 & 206.78 & 137.87 & 132.44 \\
Ockeghem & $14 / 05 / 2032$ & $15 / 05 / 2032$ & 694.30 & 689.37 & 122.39 & 0.0004 \\
\hline
\end{tabular}

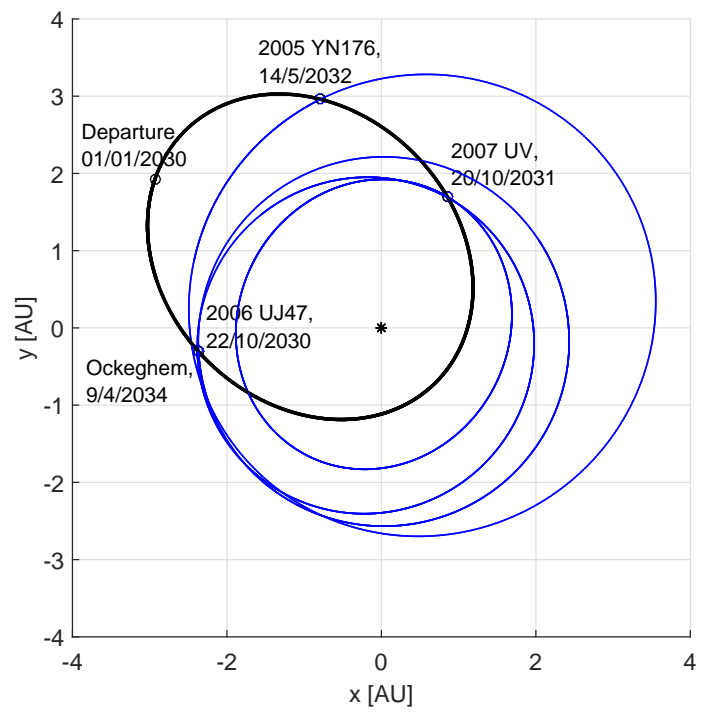

Figure 10: Selected solution for the main belt tour for Database 1.

\subsection{Transfer from Earth to the main belt}

The tour in Table 6 satisfies the 5 year requirement. The attempt now is to realise the launch and transfer to orbit $\mathcal{O} \mathcal{E}_{1}$ in less than 5 years so that the mission time is less than 10 years. Two possibilities exist for the transfer from the Earth to the selected orbit $\mathcal{O E}_{1}$, with time of transfer shorter than 5 years. The details of these options are given in Table 7 and the orbits are shown in Figure 11. In Table 7 the times $T_{L}$ and $T_{M}$ when $\Delta V_{L}$ and $\Delta V_{M}$ are 
applied, the corresponding $\Delta V$ and the orbital elements of the intermediate phasing orbit are given.

Table 7: Transfers to the orbit characterised by orbital elements $\mathcal{O} \mathcal{E}_{1}$ with transfer time shorter than 5 years.

\begin{tabular}{l|llllllll}
\hline & $T_{L}$ & $\Delta V_{L}$ & $a_{\text {int }}$ & \multirow{2}{*}{$e_{\text {int }}$} & $n_{\text {rev }}$ & $T_{M}$ & $\Delta V_{M}$ & $\Delta T$ \\
& {$[\mathrm{~km} / \mathrm{s}]$} & {$[\mathrm{AU}]$} & & & {$[\mathrm{km} / \mathrm{s}]$} & {$[$ days $]$} \\
\hline T1 & $06 / 08 / 2026$ & 2.4879 & 1.2107 & 0.1740 & 1 & $05 / 12 / 2027$ & 4.9785 & 757.44 \\
$\mathrm{~T} 2$ & $06 / 08 / 2025$ & 5.8463 & 1.7577 & 0.4311 & 1 & $05 / 12 / 2027$ & 1.6202 & 757.44 \\
\hline
\end{tabular}

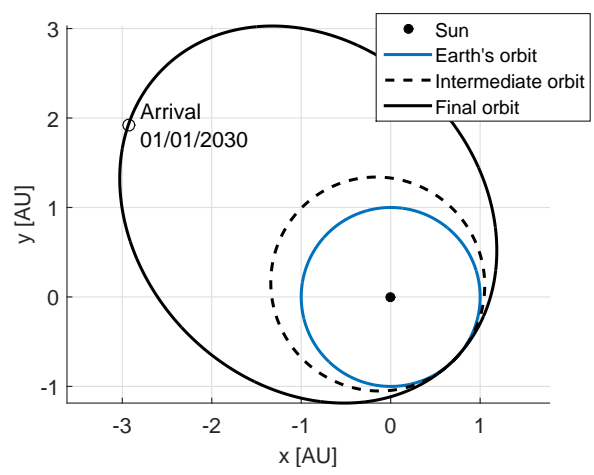

Figure 11: Transfer T1 to the orbit characterised by orbital elements $\mathcal{O E}_{1}$.

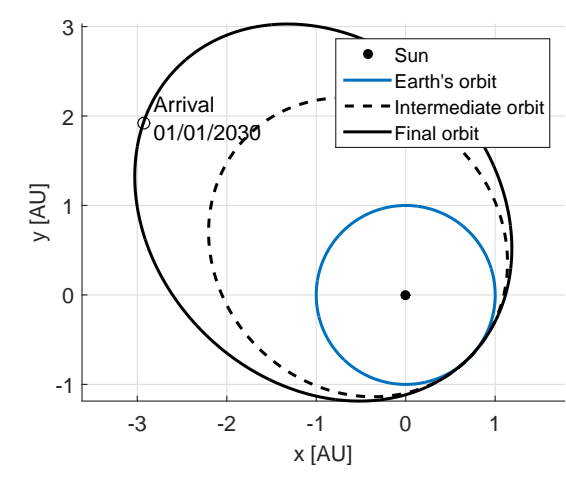

Figure 12: Transfer T2 to the orbit characterised by orbital elements $\mathcal{O E}_{1}$.

The launch for the two options T1 and T2 defined in Table 7 is investigated for two types of launchers: the Indian Space Research Organisation GSLV-D6 (Geosynchronous Satellite Launch Vehicle) ${ }^{7}$ and the European Space Agency Soyuz $^{8}$. The GTO parameters of the GLSV and Soyuz launchers are summarised in Table 8, together with their maximum payload mass in GTO, $m_{G T O}$. The quantities $h_{p, G T O}$ and $h_{a, G T O}$ are the perigee altitude and apogee altitude of the GTO orbit.

Following the method described in Section 2.4, results for the two transfer options T1 and T2 and for the two launchers are shown in Figures 13 to 15.

\footnotetext{
${ }^{7}$ Indian Space Research Organisation - http://www.isro.gov.in/launcher/gslv-d6

${ }^{8}$ Arianespace - http://www.arianespace.com/vehicle/soyuz/
} 
Table 8: Orbital elements and payload mass in GTO: GLSV and Soyuz.

\begin{tabular}{llllll}
\hline & $h_{p, G T O}[\mathrm{~km}]$ & $h_{a, G T O}[\mathrm{~km}]$ & $i_{G T O}[\mathrm{deg}]$ & $\omega_{G T O}[\mathrm{deg}]$ & $m_{G T O}[\mathrm{~kg}]$ \\
\hline GLSV & 170 & 35975 & 19 & 178 & 2330 \\
Soyuz & 250 & 35943 & 6 & 178 & 3250 \\
\hline
\end{tabular}

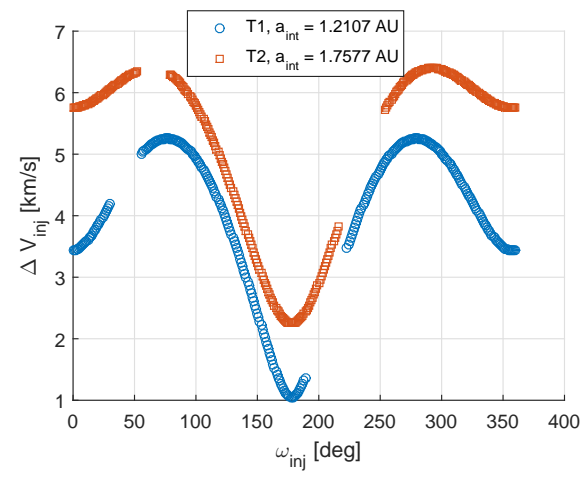

Figure 13: Variation of $\Delta V_{i n j}$ with the argument of perigee of the hyperbolic orbit for the two transfer options considered.

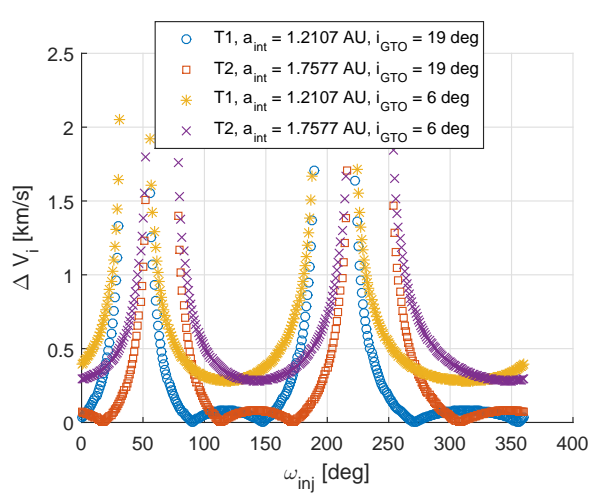

Figure 14: Variation of $\Delta V_{i}$ with the argument of perigee of the hyperbolic orbit for the two transfer options and the two launchers considered.

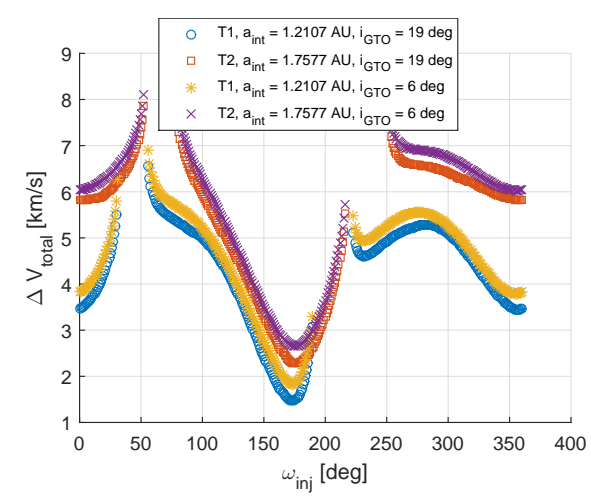

Figure 15: Variation of $\Delta V_{\text {total }}=\Delta V_{i n j}+$ $\Delta V_{i}$ with the argument of perigee of the hyperbolic orbit for the two transfer options and the two launchers considered.

Figure 13 shows the $\Delta V$ necessary for the injection into the hyperbolic orbit from the GTO, $\Delta V_{i n j}$. Notice that $\Delta V_{i n j}$ depends only on $\omega_{i n j}$. Figure 14 presents the $\Delta V$ necessary for the inclination change from the inclination of the GTO to the appropriate inclination of the hyperbolic orbit. $\Delta V_{i}$ 
depends on both the considered intermediate phasing orbit (T1 or T2) and on the launcher chosen, since the GTO of GLSV and Soyuz have different inclinations. Finally, the total $\Delta V_{\text {total }}$, given by the sum of $\Delta V_{i n j}$ and $\Delta V_{i}$, is presented in Figure 15. The minimum $\Delta V$ results are summarised in Table 9, that reports the inclination and argument of perigee of the injection hyperbolic orbit, the $\Delta V$ 's, the propellant mass and dry mass of the upper stage, $m_{\text {prop }}^{U / S}$ and $m_{d r y}^{U / S}$, the total payload mass in GTO, $m_{p l}$, and the launcher mass margin. The assumed initial wet mass of the spacecraft is $m_{0}=1000$ $\mathrm{kg}$ and the considered upper stage has $I_{s p}^{U / S}=400 \mathrm{~s}$ and propellant mass fraction $k=0.77$. Results show that, for this mass of the spacecraft, the injection into T1 could be realised using GLSV, while for T2 a Soyuz launch would be required. It is worth to recall that the reference value of $m_{0}$ chosen in this study can be changed without the need to redesign the tour and the transfer provided that the thrust is rescaled accordingly (Section 3.5). The mass margins shown in Table 9 give indication about the values of $m_{0}$ that is possible to consider for each launch option and each launcher. For example, option T1, using Soyuz, would allow to increase the mass of the spacecraft, since the mass margin is $1311.04 \mathrm{~kg}$. On the contrary, a launch with option T2 and GLSV would require a smaller spacecraft than the proposed 1000 $\mathrm{kg}$.

Table 9: Launch and injection into intermediate phasing orbit (Database 1).

\begin{tabular}{|c|c|c|c|c|c|c|c|c|c|}
\hline & $\begin{array}{l}i_{i n j} \\
\text { [deg] }\end{array}$ & $\begin{array}{l}\omega_{i n j} \\
{[\mathrm{deg}]}\end{array}$ & $\begin{array}{l}\Delta V_{i} \\
{[\mathrm{~km} / \mathrm{s}]}\end{array}$ & $\begin{array}{l}\Delta V_{i n j} \\
{[\mathrm{~km} / \mathrm{s}]}\end{array}$ & $\begin{array}{l}\Delta V_{\text {total }} \\
{[\mathrm{km} / \mathrm{s}]}\end{array}$ & $\begin{array}{l}m_{d r y}^{U S} \\
{[\mathrm{~kg}]}\end{array}$ & $\begin{array}{l}m_{\text {prop }}^{U S} \\
{[\mathrm{~kg}]}\end{array}$ & $\begin{array}{l}m_{p l} \\
{[\mathrm{~kg}]}\end{array}$ & $\begin{array}{l}\text { Margin } \\
{[\mathrm{kg}]}\end{array}$ \\
\hline T1, GLSV & 31.42 & 173 & 0.34 & 1.12 & 1.47 & 156.89 & 525.26 & 1682.16 & 647.84 \\
\hline $\mathrm{T} 2, \mathrm{C}$ & 19.89 & 176 & 0.02 & 2.26 & 2.28 & 308.46 & 1032.67 & 2341.14 & -11.14 \\
\hline T1, Soyuz & 31.28 & 173 & 0.70 & 1.13 & 1.83 & 215.96 & 722.99 & 1938.96 & 1311.04 \\
\hline T2, Soyuz & 19.84 & 176 & 0.38 & 2.27 & 2.65 & 407.03 & 1362.66 & 2769.69 & 480.31 \\
\hline
\end{tabular}

\subsection{Low-thrust optimisation}

The electric engine considered in this study has thrust magnitude $F=0.15$ $\mathrm{N}$ and specific impulse $I_{s p}=3000 \mathrm{~s}$. The initial mass of the spacecraft at launch is assumed to be $m_{0}=1000 \mathrm{~kg}$. This corresponds to a low-thrust acceleration equal to $1.510^{-4} \mathrm{~m} / \mathrm{s}^{2}$. Results for different initial mass of the spacecraft can be obtained by scaling the results presented here, under the assumption that the thrust level increases with the mass of the spacecraft, so that the acceleration is always $1.510^{-4} \mathrm{~m} / \mathrm{s}^{2}$. The low-thrust $\Delta V$ required 
to realise the transfer to $\mathcal{O} \mathcal{E}_{1}$ and the tour of the asteroids is shown in Table 10 , together with the propellant consumption $m_{\text {prop }}$ and the initial and final masses, $m_{0}$ and $m_{f}$, for the two phases of the mission (transfer to $\mathcal{O} \mathcal{E}_{1}$ and tour of the asteroids). Both the possible transfer options defined in Table 7 are considered. The low-thrust trajectories for the transfer phase T1 and T2 are shown in Figures 16 and 17, with coast arcs in gray and thrust arcs in black. The low-thrust trajectory for the tour phase corresponding to T1 is shown in Figures 18 while Figures 19 and 20 show the variation of $a$ and $e$ along the trajectory. Transfer option T2 allows for a higher final spacecraft mass (912.82 kg rather than $845.09 \mathrm{~kg}$ ) but the transfer time is one year longer and the $\Delta V$ required for the injection into orbit is higher (Table 9 ).

Table 10: $\Delta V$ and propellant consumption for the low-thrust transfer to $\mathcal{O E}_{2}$ and for the asteroids tour of Database 1.

\begin{tabular}{l|llll|llll}
\hline & \multicolumn{3}{|c}{ Transfer to $\mathcal{O E}_{1}$} & \multicolumn{4}{c}{ Asteroids tour } \\
\hline & $m_{0}$ & $\Delta V$ & $m_{\text {prop }}$ & $m_{f}$ & $m_{0}$ & $\Delta V$ & $m_{\text {prop }}$ & $m_{f}$ \\
& {$[\mathrm{~kg}]$} & {$[\mathrm{km} / \mathrm{s}]$} & {$[\mathrm{kg}]$} & {$[\mathrm{kg}]$} & {$[\mathrm{kg}]$} & {$[\mathrm{km} / \mathrm{s}]$} & {$[\mathrm{kg}]$} & {$[\mathrm{kg}]$} \\
\hline $\mathrm{T} 1$ & 1000 & 4.0604 & 129 & 871 & 871 & 0.8881 & 25.91 & 845.09 \\
$\mathrm{~T} 2$ & 1000 & 1.9582 & 64.43 & 935.57 & 935.57 & 0.7236 & 22.75 & 912.82 \\
\hline
\end{tabular}

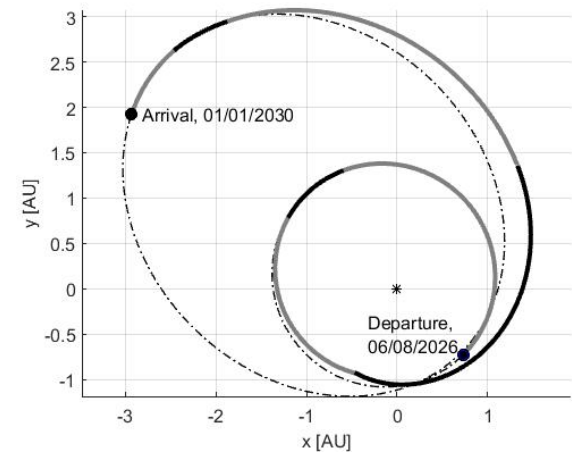

Figure 16: Low-thrust transfer trajectory to $\mathcal{O} \mathcal{E}_{1}$, option $\mathrm{T} 1$.

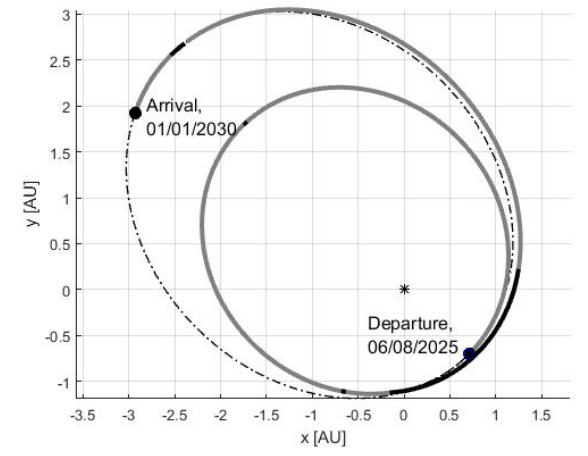

Figure 17: Low-thrust transfer trajectory to $\mathcal{O} \mathcal{E}_{1}$, option T2. 


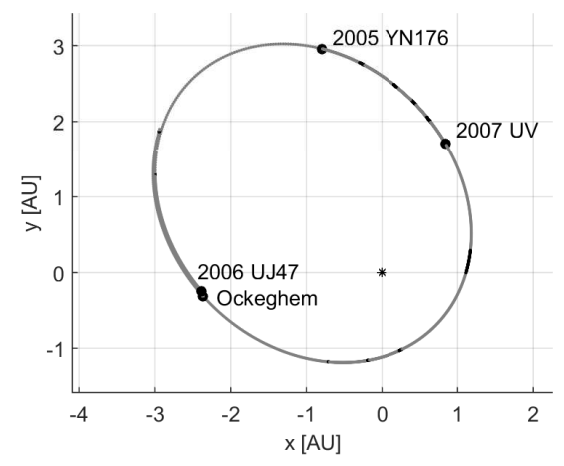

Figure 18: Low-thrust trajectory for the tour of the asteroids of Database 1.
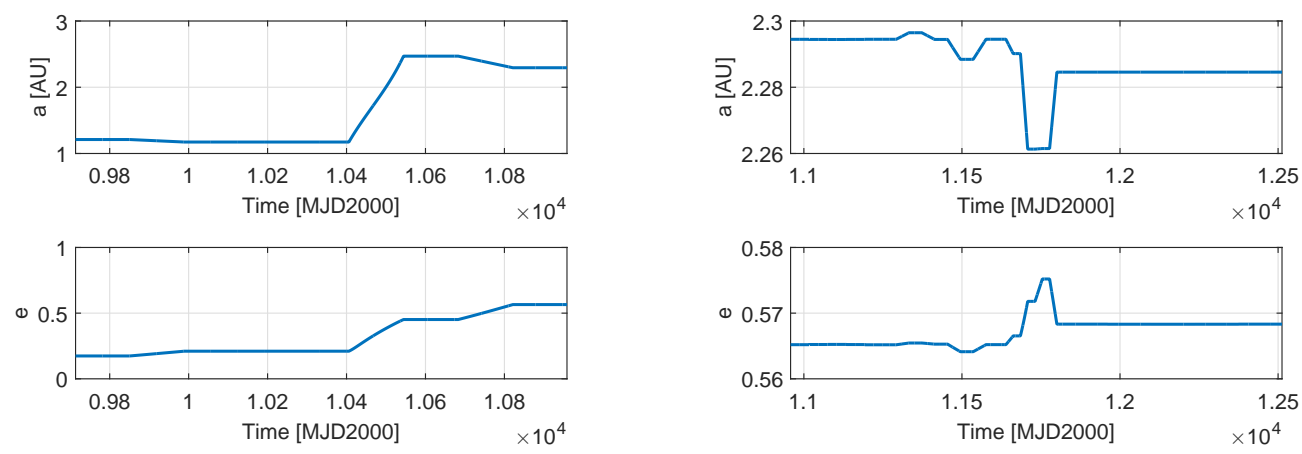

Figure 19: Variation of semimajor axis and eccentricity during the low-thrust transfer to $\mathcal{O} \mathcal{E}_{1}$.

Figure 20: Variation of semimajor axis and eccentricity during the low-thrust transfertour of the asteroids of Database 1.

\section{Results Database $1+2$}

Results from Section 3 show that the maximum number of scientifically interesting asteroids that can be visited is four. The main belt, however, houses more than 641,933 objects; with such a large number of objects, additional asteroids of reduced scientific interest might be visited while travelling between two asteroids in Database 1. In order to study this scenario, the two Databases 1 and 2 were combined and new sequences were generated. This section presents the results of this analysis.

\subsection{Minimum Orbit Intersection Distance}

As before, the MOID is computed between all the asteroids in the combined database and different orbits of the spacecraft identified by the orbital 
elements in Table 11.

Table 11: Orbital elements of the different possible initial orbits of the spacecraft used for the computation of the MOID.

\begin{tabular}{lllll}
\hline$r_{p}[\mathrm{AU}]$ & $r_{a}[\mathrm{AU}]$ & $i[\mathrm{deg}]$ & $\Omega[\mathrm{deg}]$ & $\omega[\mathrm{deg}]$ \\
\hline 1 & {$[1.86,2.46]$} & 0 & 0 & {$[0,360]$} \\
\hline
\end{tabular}

Figure 21 shows, for each analysed value of the aphelion $r_{a}$ and for different values of $\omega$, the number of asteroids with $d<0.01 \mathrm{AU}$ with respect the orbit of the spacecraft. As before, the higher the aphelion the greater the number of asteroids with $d<0.01 \mathrm{AU}$. This is true in the range of $r_{a}$ considered in this study. We then apply the phasing process presented in Subsection 2.1 to further reduce the shortlist. Figure 22 shows the number of asteroids that respect the condition in Equation 1, for different values of $M_{0}$ and for the value of $\omega$ giving the maximum number of asteroids with $d<0.01 \mathrm{AU}$; $\delta=1$ in this case. The number of asteroids with $d<0.01 \mathrm{AU}$ and phasing condition satisfied can be as high as 82 , when $r_{a}=2.46$ AU. However, only transfers with a total $\Delta V$ lower than $\Delta V_{\max }$ are considered. The sequence of asteroids that satisfy $\Delta V<\Delta V_{\max }$ are presented in the next section.

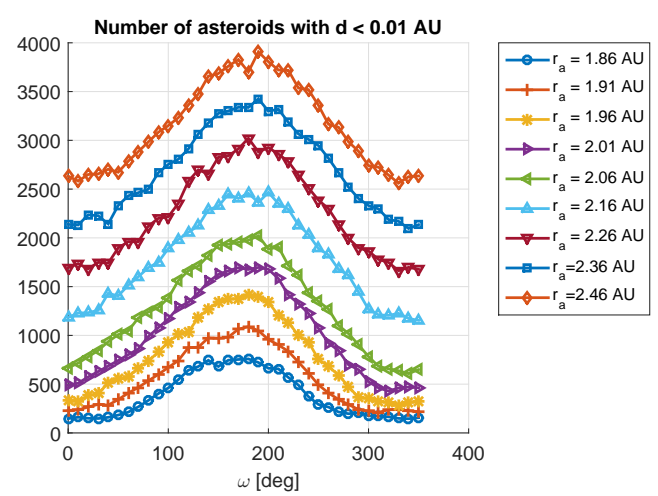

Figure 21: Number of asteroids with $d<$ $0.01 \mathrm{AU}$ for different initial orbit of the spacecraft, identified by their aphelion radius $r_{a}$.

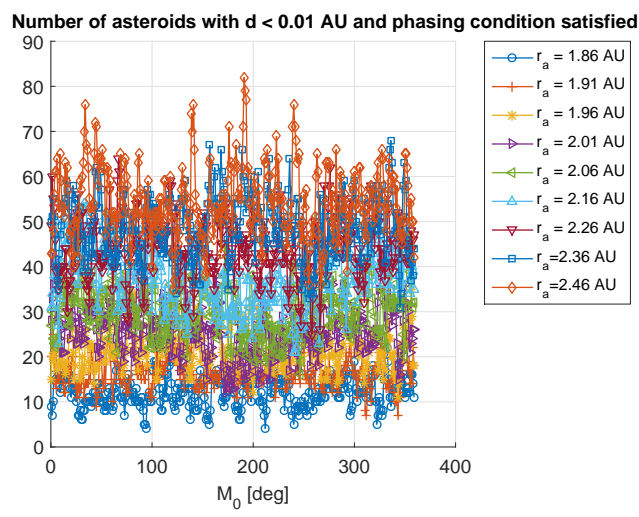

Figure 22: Number of asteroids with $d<0.01 \mathrm{AU}$ and phasing condition for asteroids encounter (Equation 1) satisfied. 


\subsection{Study of the possible sequences of asteroids}

Figure 23 shows the $\Delta V$ required for the tour of the asteroids, as a function of the number $N$ of visited objects. The initial orbit of the spacecraft has $r_{a}=1.86 \mathrm{AU}$ and $\omega=180 \mathrm{deg}$ and the maximum mission cost is $\Delta V_{\max }=$ $1 \mathrm{~km} / \mathrm{s}$. Different values of the angle $\delta$ are considered, from $\delta=0.1 \mathrm{deg}$ to $\delta=1 \mathrm{deg}$. The values of $\delta$ used in this section are different from the one used in Section 3. The dimension of the considered database of asteroids $(\sim$ $100,000$ vs. $\sim 400)$ results in unmanageable computational time and amount of data generated when $\delta$ is larger than the value used here. Figure 23 collects the results for all the possible values of $M_{0}$ from 1 to $359 \mathrm{deg}$, at steps of 1 deg and, for each value of $N$, only the first 1000 best solution (the ones with lower $\Delta V)$ are shown.
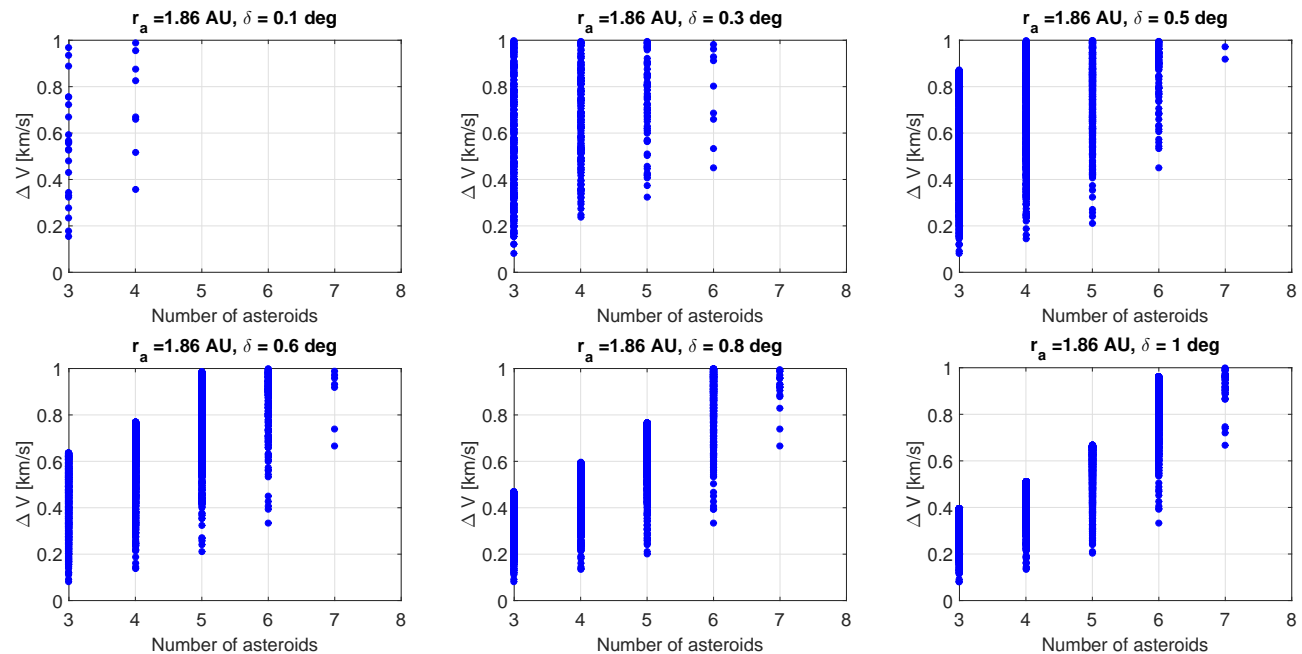

Figure 23: Relation between $\Delta V$ and number of visited asteroids for orbit with $r_{a}=1.86$ AU and different values of $\delta$.

Results from Figure 23 show that higher values of $\delta$ allows one to find solutions with a longer list of asteroids, while still satisfying the condition $\Delta V<\Delta V_{\max }$. The maximum value of $N$ is indeed 4 for $\delta=0.1 \mathrm{deg}$ and $N=7$ for $\delta=1 \mathrm{deg}$. Figure 24 shows the relation between $\Delta V$ and number of visited asteroids for orbits with different values of $r_{a}$, as defined in Table 11 , and different values of $\delta$. The value of $\omega$ for each orbit is the one that allows one to visit the maximum possible number of asteroids for that $r_{a}$. As $r_{a}$ increases, the maximum number of asteroids that can be visited increases 
from 8 , for $r_{a}=1.86 \mathrm{AU}$, to 11 for $r_{a}=2.46 \mathrm{AU}$ and the $\Delta V$ associated to a given number of asteroids $N$ decreases.
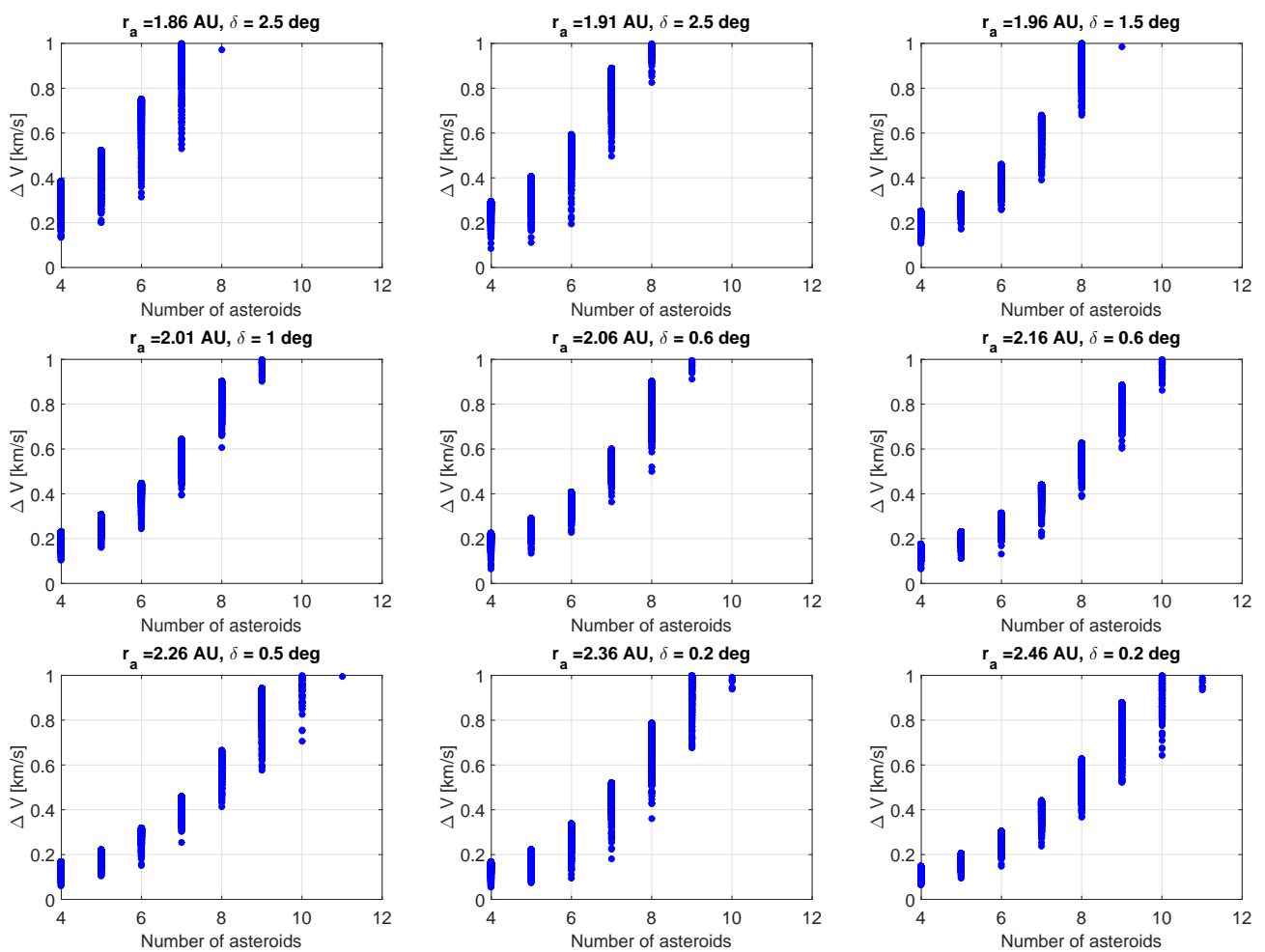

Figure 24: Relation between $\Delta V$ and number of visited asteroids for orbits with different $r_{a}$ and for different values of $\delta$.

Figure 24 shows that the maximum number of visited asteroids, $N=11$, can be obtained using an orbit with $r_{a}=2.26 \mathrm{AU}$ or $r_{a}=2.46 \mathrm{AU}$. For $r_{a}=2.26 \mathrm{AU}$ and $\delta=0.5 \mathrm{deg}$, the binary vector $\mathbf{b}$ composed of 0 's and 1's has a length depending on the value of $M_{0}$ ( $M_{0}$ ranges from 1 to $\left.359 \mathrm{deg}\right)$. The minimum length of $\mathbf{b}$ is $n=4$ and the maximum length is $n=31$. The BPA has, therefore, to handle a maximum of $2^{31}$ sequences (Section 2.2). The total computation time, for all the values of $M_{0}$ ranging from 1 to $359 \mathrm{deg}$, is 82 minutes on a Intel(R) Core(TM) i7-3770 CPU 3.4 GHz and 8 GB RAM using Matlab R2015a. In the following the solution characterised by $r_{a}=2.26$ $\mathrm{AU}$ is analysed in more detail. Figure 25 shows the maximum number of asteroids that is possible to visit with maximum tour cost $\Delta V_{\max }=1 \mathrm{~km} / \mathrm{s}$ for different initial dates from December 2029 to January 2030 and $r_{a}=2.26$ 
AU. The best results are obtained with initial date $01 / 01 / 2030$, the one chosen for this study.

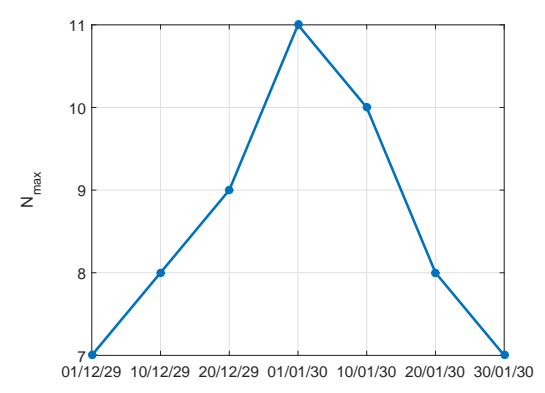

Figure 25: Maximum number of visited asteroids for $\Delta V_{\max }=1 \mathrm{~km} / \mathrm{s}$ and different initial date for the tour (dates in $\mathrm{dd} / \mathrm{mm} / \mathrm{yy}$ ).

Since MP-AIDEA can reduce the $\Delta V$ cost of the mission, for $r_{a}=2.26$ AU the binary tree for the generation of the possible sequences of asteroids is run also for $\Delta V_{\max }=2 \mathrm{~km} / \mathrm{s}$. The aim is to obtain, after optimisation, $\Delta V_{\text {opt }}<1 \mathrm{~km} / \mathrm{s}$ with $N \geq 11$.

Results show that, within the limit of $\Delta V_{\max }=2 \mathrm{~km} / \mathrm{s}$, the maximum number of asteroids that can be visited is $N=14$.

However, by inspecting all the sequences, one can see that:

- For $N=14$, only two different sequences are identified. They do not include any of the scientifically interesting asteroids in Database 1.

- For $N=13,262$ possible sequences are found, none of which include asteroids from Database 1.

- For $N=12,5764$ sequences are found, 29 of which included 1 or 2 asteroids from Database 1. Among the 29 solutions with asteroids from Database 1, the one with lowest cost and two scientifically interesting asteroids has a $\Delta V=1.7574 \mathrm{~km} / \mathrm{s}$.

- For $N=11,84606$ possible sequences are found, out of which 2109 include 1 or 2 asteroids from Database 1. The solution with lowest $\Delta V$ and 2 scientifically interesting asteroids has a cost of $1.1865 \mathrm{~km} / \mathrm{s}$. 


\subsection{Optimisation of the sequence of asteroids}

The solutions with $N=11$ and $N=12$ that include 2 asteroids from Database 1 and with lowest $\Delta V$ are further optimised using MP-AIDEA. The settings of the optimisation problem and the boundaries of the search space are the same ones used for Database 1. After optimisation, the solution characterised by $N=12$ and $\Delta V=1.7574 \mathrm{~km} / \mathrm{s}$ gives an optimised cost of $\Delta V_{\text {opt }}=1.3 \mathrm{~km} / \mathrm{s}$. The solution characterised by $N=11$ and $\Delta V=1.1865$ gives $\Delta V_{\text {opt }}=0.7613 \mathrm{~km} / \mathrm{s}$. Since in this case $\Delta V<1 \mathrm{~km} / \mathrm{s}$, this solution is the one selected for further analysis. Details of the asteroids visited, times of encounters and $\Delta V$ are given in Table 12, while a graphical representation is given in Figure 26 and Figure 27.

The scientifically interesting asteroids are represented in bold in Table 12 . Both 2003 QS31 and 2110 Moore-Sitterly are asteroid pairs.

Table 12: Selected solution for the main belt tour for Database 1+2. Interesting asteroids from Database 1 are shown in bold.

\begin{tabular}{lllllll}
\hline $\begin{array}{l}\text { Targeted } \\
\text { Asteroid }\end{array}$ & \multirow{2}{*}{ Dep. Date } & $\begin{array}{l}\text { Optimised } \\
\text { Dep. Date }\end{array}$ & $\begin{array}{l}\text { ToF } \\
\text { [days] }\end{array}$ & $\begin{array}{l}\text { Opt. ToF } \\
{[\text { days }]}\end{array}$ & $\begin{array}{l}\Delta V \\
{[\mathrm{~m} / \mathrm{s}]}\end{array}$ & $\begin{array}{l}\text { Opt. } \Delta V \\
{[\mathrm{~m} / \mathrm{s}]}\end{array}$ \\
\hline 2012 DW5 & $1 / 1 / 2030$ & $1 / 1 / 2030$ & 78.77 & 81.37 & 80.42 & 62.64 \\
2005 QM95 & $20 / 3 / 2030$ & $23 / 3 / 2030$ & 148.27 & 145.02 & 240.41 & 16.12 \\
2007 UJ78 & $16 / 8 / 2030$ & $15 / 8 / 2030$ & 119.12 & 119.92 & 216.58 & 113.86 \\
2003 QS31 & $13 / 12 / 2030$ & $13 / 12 / 2030$ & 392.10 & 392.35 & 101.55 & 108.34 \\
2001 QY152 & $9 / 1 / 2032$ & $9 / 1 / 2032$ & 105.36 & 105.25 & 51.17 & 37.07 \\
2009 HL17 & $23 / 4 / 2032$ & $23 / 4 / 2032$ & 92.23 & 92.34 & 90.53 & 73.30 \\
2005 SF9 & $24 / 7 / 2032$ & $25 / 7 / 2032$ & 143.38 & 143.43 & 94.41 & 74.04 \\
Moore-Sitterly & $15 / 12 / 2032$ & $15 / 12 / 2032$ & 409.26 & 409.08 & 35.33 & 13.92 \\
2000 QL & $28 / 1 / 2034$ & $28 / 1 / 2034$ & 64.72 & 64.88 & 106.24 & 107.09 \\
2000 YU15 & $3 / 4 / 2034$ & $3 / 4 / 2034$ & 264.52 & 264.27 & 147.52 & 123.63 \\
2000 VT44 & $23 / 12 / 2034$ & $23 / 12 / 2034$ & 59.97 & 59.82 & 22.40 & 31.28 \\
\hline
\end{tabular}

The initial orbit of the spacecraft in the main belt has optimised orbital elements: $\mathcal{O E}_{1+2}=\{a=1.6299 \mathrm{AU}, e=0.3826, i=0 \mathrm{deg}, \Omega=0 \mathrm{deg}, \omega=$ $180.3330 \mathrm{deg}, M_{0}=102.36 \mathrm{deg}, t_{0}=10958.5$ MJD2000 $\}$.

\subsection{Transfer from Earth to the main belt}

Two possibilities exist for the transfer to the orbit $\mathcal{O E}_{1+2}$ with time of flight shorter than 5 years. These are presented in Table 13 and Figures 28 and 29 . 


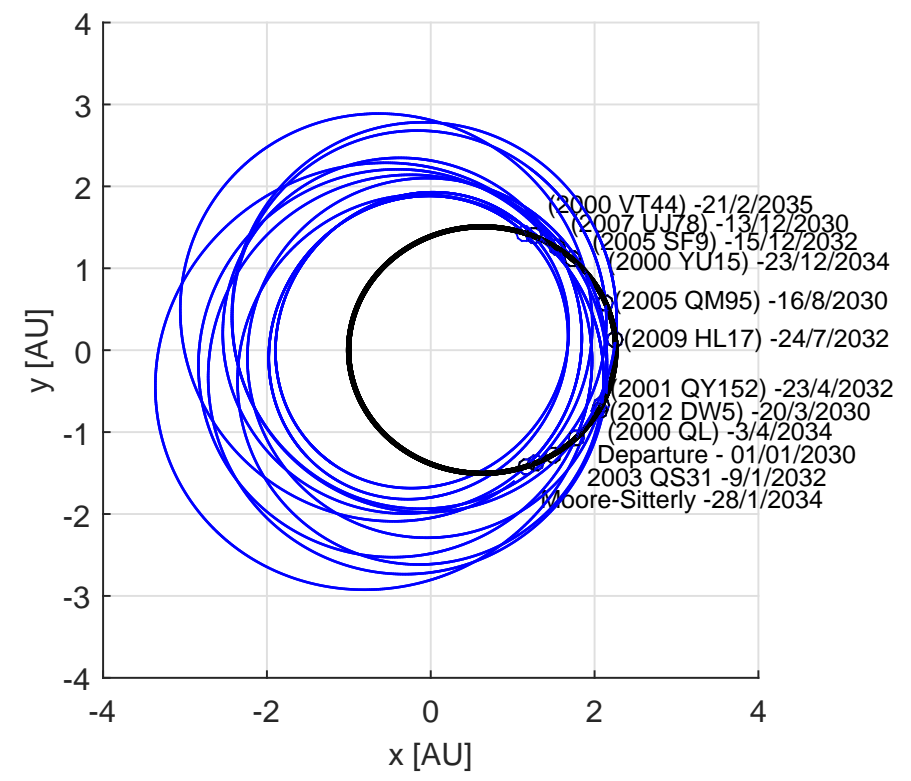

Figure 26: Selected solution for the main belt tour for Database $1+2$.

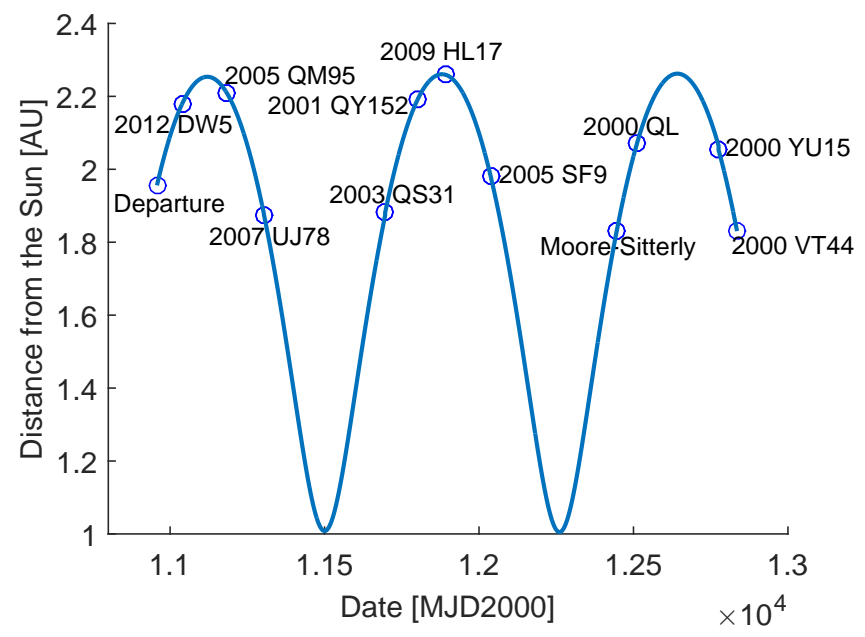

Figure 27: Distance of the spacecraft from the Sun for the selected solution for the main belt tour for Database $1+2$.

Following the method described in Section 2.4, results for the two transfer options T1 and T2 and for the two launchers are shown in Figures 30 to 32. 
Table 13: Transfers to the orbit characterised by orbital elements $\mathcal{O} \mathcal{E}_{1+2}$ with transfer time shorter than 5 years.

\begin{tabular}{l|llllllll}
\hline & $T_{L}$ & $\Delta V_{L}$ & $a_{\text {int }}$ & \multirow{2}{*}{$e_{\text {int }}$} & $n_{\text {rev }}$ & $T_{M}$ & $\Delta V_{M}$ & $\Delta T$ \\
& {$[\mathrm{~km} / \mathrm{s}]$} & {$[\mathrm{AU}]$} & & & {$[\mathrm{km} / \mathrm{s}]$} & {$[$ days $]$} \\
\hline T1 & $21 / 03 / 2028$ & 1.5935 & 1.1234 & 0.1099 & 1 & $29 / 05 / 2029$ & 3.6929 & 216.09 \\
$\mathrm{~T} 2$ & $21 / 03 / 2026$ & 3.7491 & 1.3653 & 0.2676 & 2 & $29 / 05 / 2029$ & 1.5374 & 216.09 \\
\hline
\end{tabular}

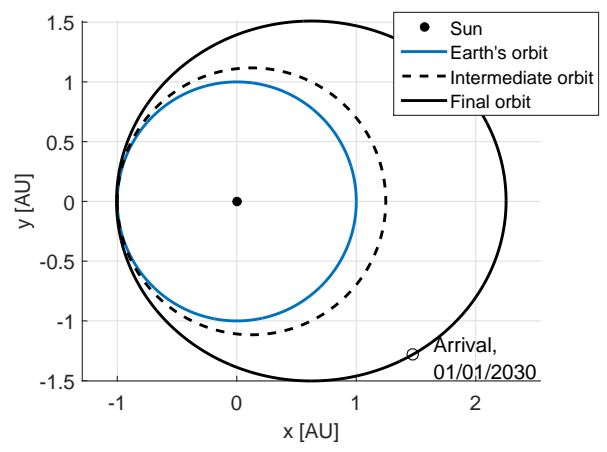

Figure 28: Orbits for transfer option T1 from Earth to orbit $\mathcal{O} \mathcal{E}_{1+2}$.

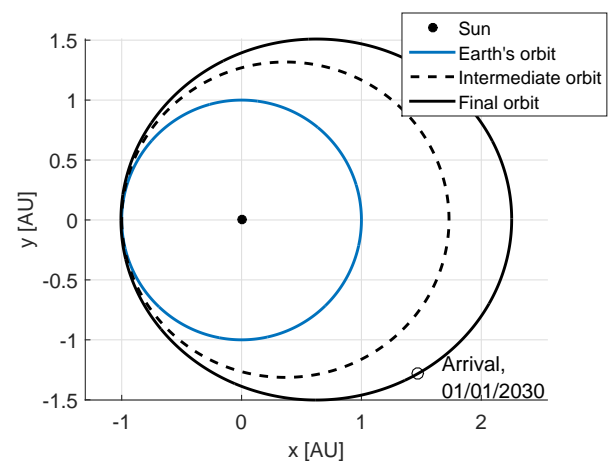

Figure 29: Orbits for transfer option T2 from Earth to orbit $\mathcal{O E}_{1+2}$.

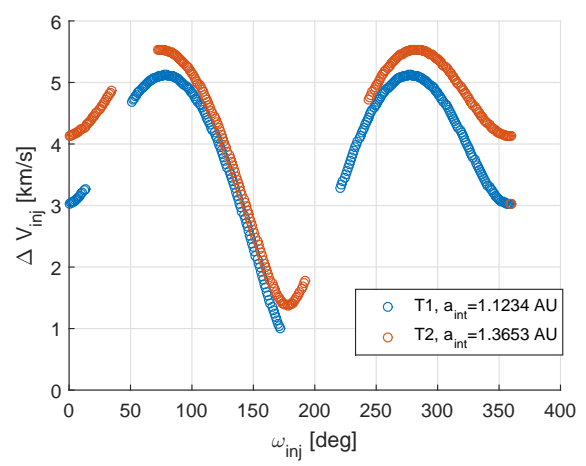

Figure 30: Variation of $\Delta V_{i n j}$ with the argument of perigee of the hyperbolic orbit for the two transfer options considered.

The minimum $\Delta V$ results are summarised in Table 14. Both GSLV and Soyuz can be used to inject the spacecraft and upper stage into GTO, with Soyuz allowing for a larger mass margin. 


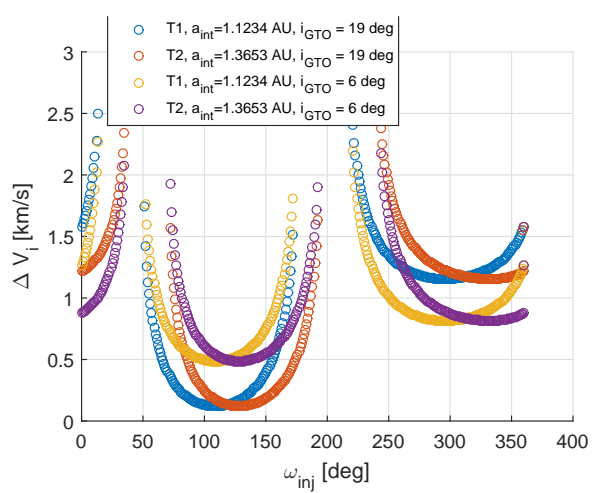

Figure 31: Variation of $\Delta V_{i}$ with the argument of perigee of the hyperbolic orbit for the two transfer options and the two launchers considered.

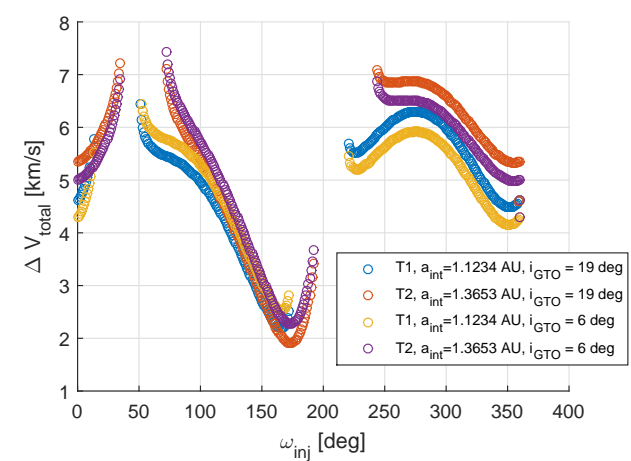

Figure 32: Variation of $\Delta V_{\text {total }}=\Delta V_{i n j}+$ $\Delta V_{i}$ with the argument of perigee of the hyperbolic orbit for the two transfer options and the two launchers considered.

Table 14: Injection into intermediate phasing orbit (Database $1+2)$.

\begin{tabular}{|c|c|c|c|c|c|c|c|c|c|}
\hline & $\begin{array}{l}i_{i n j} \\
{[\mathrm{deg}]}\end{array}$ & $\begin{array}{l}\omega_{i n j} \\
{[\mathrm{deg}]}\end{array}$ & $\begin{array}{l}\Delta V_{i} \\
{[\mathrm{~km} / \mathrm{s}]}\end{array}$ & $\begin{array}{l}\Delta V_{i n j} \\
{[\mathrm{~km} / \mathrm{s}]}\end{array}$ & $\begin{array}{l}\Delta V_{\text {total }} \\
{[\mathrm{km} / \mathrm{s}]}\end{array}$ & $\begin{array}{l}m_{d r y}^{U S} \\
{[\mathrm{~kg}]}\end{array}$ & $\begin{array}{l}m_{\text {prop }}^{U S} \\
{[\mathrm{~kg}]}\end{array}$ & $\begin{array}{l}m_{p l} \\
{[\mathrm{~kg}]}\end{array}$ & $\begin{array}{l}\text { Margin } \\
{[\mathrm{kg}]}\end{array}$ \\
\hline $\mathrm{T} 1$, & 51.94 & 166 & 0.91 & 1.29 & 2.20 & 290.10 & 971.21 & 2261.31 & 68.69 \\
\hline T2, GLSV & 35.93 & 173 & 0.47 & 1.44 & 1.91 & 230.82 & 772.74 & 2003.56 & 326.44 \\
\hline $\mathrm{T} 1$, & 51.73 & 166 & 1.25 & 1.29 & 2.54 & 374.21 & 1252.77 & 2626.98 & 623.02 \\
\hline T2, Soyuz & 35.78 & 173 & 0.83 & 1.44 & 2.27 & 305.99 & 1024.42 & 2330.42 & 919.58 \\
\hline
\end{tabular}

\subsection{Low-thrust optimisation}

The $\Delta V$ required to realise the low-thrust transfer to $\mathcal{O} \mathcal{E}_{1+2}$ and the tour of the asteroids is shown in Table 15, together with the propellant consumption $m_{\text {prop }}$ and the initial and final mass, $m_{0}$ and $m_{f}$, for the two phases of the mission (transfer to $\mathcal{O} \mathcal{E}_{1}$ and tour of the asteroids). Both the possible transfer options defined in Table 13 are evaluated.

The low-thrust trajectories for the transfer phases T1 and T2 are shown in Figure 33 and 34. The low-thrust trajectory for the asteroid tour phase of option $\mathrm{T} 1$ and the corresponding variation of $a$ and $e$ are shown in Figure 35 and Figures 36 and 37 . Table 15 shows that transfer option T2 results in a higher final mass of the spacecraft $(875.40 \mathrm{~kg})$ than option T1. Option T2 has also a lower $\Delta V_{\text {total }}$ than T1 (Table 14), but the transfer time is 2 years longer. 
Table 15: $\Delta V$ and propellant consumption for the low-thrust transfer to $\mathcal{O E}_{1+2}$ and tour of Database $1+2$.

\begin{tabular}{l|llll|llll}
\hline & \multicolumn{4}{|c|}{ Transfer to $\mathcal{O E}_{1+2}$} & \multicolumn{4}{c}{ Asteroids tour } \\
\hline & $m_{0}$ & $\Delta V$ & $m_{\text {prop }}$ & $m_{f}$ & $m_{0}$ & $\Delta V$ & $m_{\text {prop }}$ & $m_{f}$ \\
& {$[\mathrm{~kg}]$} & {$[\mathrm{km} / \mathrm{s}]$} & {$[\mathrm{kg}]$} & {$[\mathrm{kg}]$} & {$[\mathrm{kg}]$} & {$[\mathrm{km} / \mathrm{s}]$} & {$[\mathrm{kg}]$} & {$[\mathrm{kg}]$} \\
\hline $\mathrm{T} 1$ & 1000 & 4.1345 & 131.19 & 868.81 & 868.81 & 2.9132 & 81.96 & 786.85 \\
$\mathrm{~T} 2$ & 1000 & 1.4541 & 48.25 & 951.75 & 951.75 & 2.4584 & 76.35 & 875.40 \\
\hline
\end{tabular}

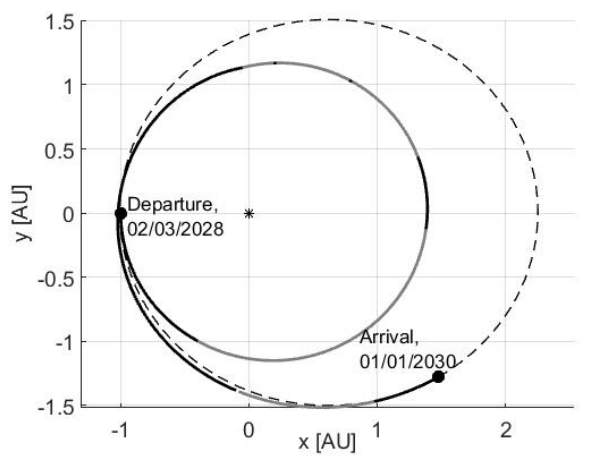

Figure 33: Low-thrust transfer trajectory to $\mathcal{O} \mathcal{E}_{1+2}$, option $\mathrm{T} 1$.

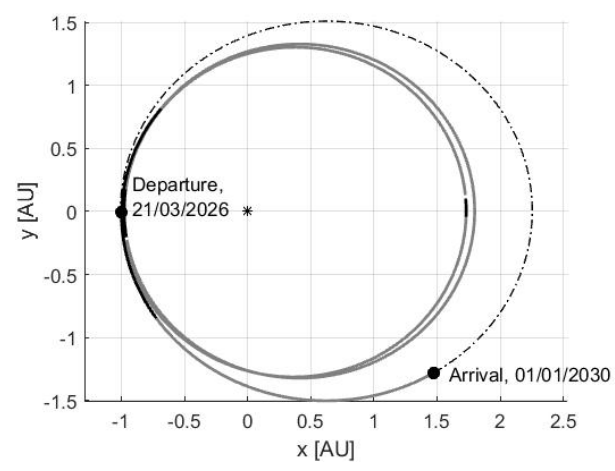

Figure 34: Low-thrust transfer trajectory to $\mathcal{O} \mathcal{E}_{1+2}$, option $\mathrm{T} 2$.

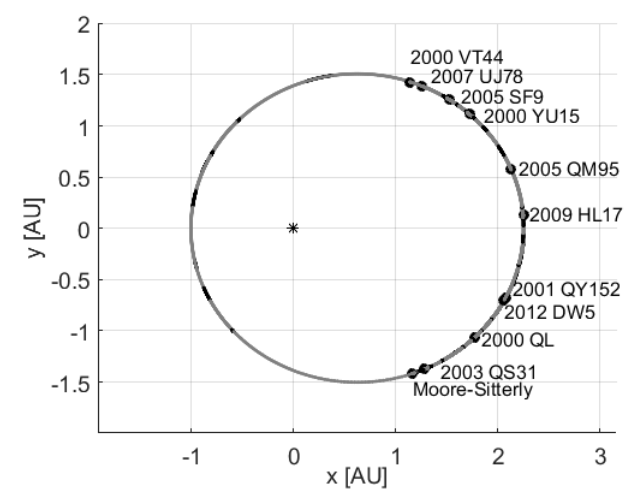

Figure 35: Low-thrust trajectory for the tour of the asteroids of Database $1+2$.

\subsection{Summary of the main mission options for Database 1 and $1+2$}

The main results of the mission options for Database 1 and Database 1 +2 are summarised in Table 16. The table gives the time of flight for the 

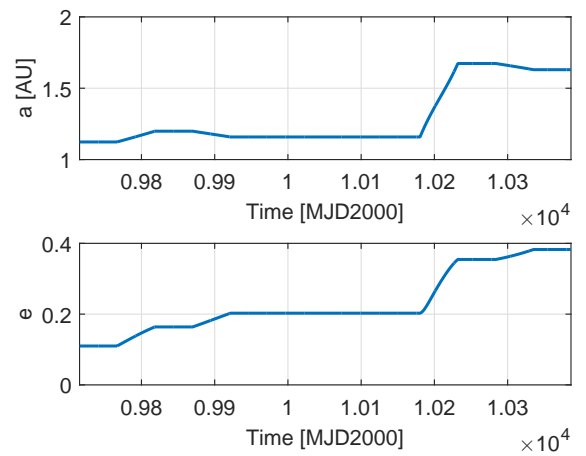

Figure 36: Variation of semimajor axis and eccentricity during the low-thrust transfer to $\mathcal{O} \mathcal{E}_{1+2}$.
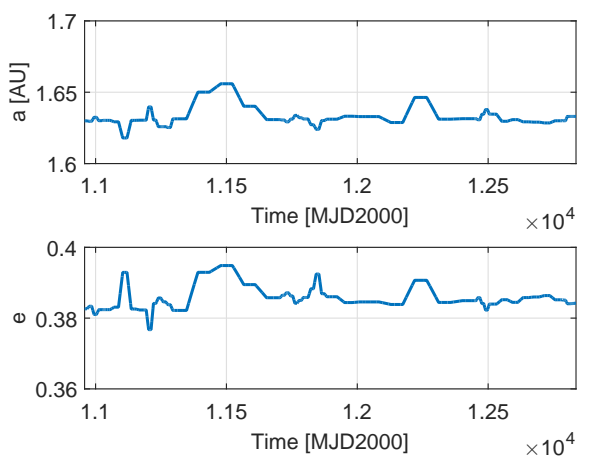

Figure 37: Variation of semimajor axis and eccentricity during the low-thrust tour of the selected objects of Database $1+2$.

transfer from Earth to $\mathcal{O} \mathcal{E}_{1}$ and $\mathcal{O} \mathcal{E}_{1+2}$, the time of flight of the tour of the asteroids, the total $\Delta V$ to be provided by the low-thrust engine and the final mass of the spacecraft, when an initial $1000 \mathrm{~kg}$ spacecraft is considered. The two considered transfer options, T1 and T2, are presented. In both cases, T2 is characterised by longer transfer time but lower $\Delta V$ and, therefore, higher final mass.

Table 16: Summary of the main mission options for Database 1 and Database $1+2$.

\begin{tabular}{l|llll}
\hline \multicolumn{5}{c}{ Database 1 (4 visited asteroids) } \\
\hline & Transfer & Tour & Total & $m_{f}$ \\
& ToF $[$ days $]$ & ToF $[$ days $]$ & $\Delta V[\mathrm{~km} / \mathrm{s}]$ & {$[\mathrm{kg}]$} \\
\hline T1 & 1244 & 865 & 4.9485 & 845.09 \\
T2 & 1609 & 865 & 2.6818 & 912.82 \\
\hline \multicolumn{5}{c}{ Database $1+2(11$ visited asteroids $)$} \\
\hline & Transfer & Tour & Total & $m_{f}$ \\
& $T o F[$ days $]$ & $T o F[$ days $]$ & $\Delta V[\mathrm{~km} / \mathrm{s}]$ & {$[\mathrm{kg}]$} \\
\hline T1 & 651 & 1817 & 7.0477 & 786.85 \\
T2 & 1382 & 1817 & 3.9125 & 875.40 \\
\hline
\end{tabular}




\section{Conclusions}

The paper presented some preliminary results for a possible low-thrust tour of the main belt. A particular strategy was investigated that attempts to maximise the number of flyby's, with interesting asteroids, by traversing the main belt with a heliocentric elliptical orbit with perihelion at the Earth and aphelion at, or beyond, the main belt region. It was shown that the coupling of a binary decision tree with a novel transcription method, for optimal control, based on an asymptotic expansions of the accelerated Keplerian motion provided a range of interesting solutions to this trajectory design problem.

The analysis on the database of targets with particular scientific relevance showed that, with an estimated maximum $\Delta V$ budget of $1 \mathrm{~km} / \mathrm{s}$ for the the asteroid tour, 4 scientifically interesting asteroids can be visited in about five years. If the database of scientifically interesting asteroids is expanded with more than 100,000 additional objects, a total of 11 asteroids could be visited with the same upper limits on mission time and $\Delta V$ budget, but among them only 2, at most, belong to the database of scientifically interesting targets. The paper also demonstrated that with low-thrust propulsion, the tour could be completed with approximately $213 \mathrm{~kg}$ of propellant, within a mission time of less than 7 years, and a launch with the GLSV launcher, leaving a mass margin of $68 \mathrm{~kg}$. Higher mass margins are possible allowing for longer transfer times to the main belt or using the Soyuz launcher.

It was noted that by increasing the $\delta$ tolerance on the phasing and relaxing the constraint on the estimated $\Delta V$ even longer sequences are possible with an optimised $\Delta V$ that might make the mission possible with heavier launchers. Furthermore, the launch and transfer strategy in this preliminary analysis do not include any swing-by. It is expected that a single or double fly-by of Mars could improve the number of visited asteroids, as the work of previous authors suggests. This will be the object of a future study.

\section{Acknowledgments}

This research was partially funded by Airbus Defence and Space and partially by the FP7 MSCA ITN Stardust. The authors would like to thank Dr Pau Sanchez, at Cranfield University, the CASTAway team, and Mr Stephen Kemble, at Airbus DS, for their support and advice. 


\section{References}

Alemany, K. \& Braun, R. D., Survey of Global Optimization Methods for Low-Thrust, Multiple Asteroid Tour Missions, Proceedings of the 17th AAS/AIAA Space Flight Mechanics Meeting, January 28 - February 1, 2007, Sedona, Arizona, Paper AAS 07-211.

Bonanno, C., An analytical approximation for the MOID and its consequences, Astronomy and Astrophysics, Vol. 360, pp. 411-416, 2000

Bottke, W. F. Jr., Jedicke, R., Morbidelli, A., et al. Understanding the Distribution of Near-Earth Asteroids, Science, Vol. 228, Issue 5474, pp. 21902194, 2000. doi: 10.1126/science. 288.5474.2190

Bowles, N., Snodgrass, C., Gibbings, A. et al. CASTAway: An Asteroid Main Belt Tour and Survey, Advances in Space Research, In Press, November 2017. doi: $10.1016 / j$.asr. 2017.10.021

Chen, Y., Baoyin, H. \& Junfeng, L. Accessibility of Main-Belt Asteroids via Gravity Assist, Journal of Guidance, Control, and Dynamics, Vol. 37, No. 2, pp. 623-632, 2014. doi: 10.2514/1.58935

Di Carlo, M., Romero Martin, J. M. \& Vasile, M., CAMELOT: Computational-Analytical Multi-fidElity Low-thrust Optimisation Toolbox, CEAS Space Journal 2017, pp. 1-12, September 2017, doi: 10.1007/ s12567-017-0172-6

Di Carlo, M., Romero Martin, J. M., Ortiz Gomez, N. \& Vasile, M., Optimised Low-Thrust Mission to the Atira Asteroids, Advances in Space Research, Vol. 59, Issue 7, 1 April 2017, pp. 1724-1739 doi: 10.1016/j. asr. 2017.01.009

Di Carlo, M., Vasile, M. \& Minisci, E., Multi-Population Adaptive Inflationary Differential Evolution Algorithm, Proceedings of the 2015 IEEE Congress on Evolutionary Computation, 25-28 May 2015, Sendai, Japan. doi: 10.1109/CEC. 2015.7256950

Grigoriev, I. S. \& Zapletin, M. P., Choosing promising sequences of asteroids, Automation and Remote Control, Vol. 74, Issue 8, pp. 1284-1296, 2013. doi: $10.1134 /$ S0005117913080055 
Gronchi, G. F., On the stationary points of the squared distance between two ellipses with a common focus, SIAM Journal on Scientific Computing, Vol. 24, pp. 61-80, 2002. doi: 10.1137/S1064827500374170

Gronchi, G. F., An algebraic method to compute the critical points of the distance function between two Keplerian orbits, Celestial Mechanics and Dynamical Astronomy, Vol. 93, pp. 295-329, 2005. doi: 10.1007/ s10569-005-1623-5

Kemble, S., Interplanetary Missions Analysis and Design, Springer Science \& Business Media, Berlin, Germany, 2006

Lantoine, G. and Russell, R. P., A Hybrid Differential Dynamic Programming Algorithm for Constrained Optimal Control Problems. Part 2: Application, Journal of Optimization Theory and Applications, Vol. 154, Issue 2, pp. 418-442, 2012. doi: 10.1007/s10957-012-0038-1

Millis, R. L., Wasserman, L. H, Franz, O.G. et al., The size, shape, density, and albedo of Ceres from its occulation of $\mathrm{BD}+8471$, Icarus, Vol. 72, Issue 3, pp. 507-518, 1987. doi: 10.1016/0019-1035(87)90048-0

Minton, D. A., Dynamical History of the Asteroid Belt and Implications for Terrestrial Planet Bombardment, PhD Thesis, University of Arizona, 2009, http://www.lpl.arizona.edu/ daminton/Minton_dissertation.pdf

Oh, D. Y. \& Landau, D., A Simple Semi-Analytic Model for Optimum Specific Impulse Interplanetary Low Thrust Trajectories, Proceedings of the 32nd International Electric Propulsion Conference, 11-15 September 2011, Wiesbaden, Germany, http://erps . spacegrant.org/uploads/images/images/iepc_ articledownload_1988-2007/2011index/IEPC-2011-010.pdf

Olympio, J. T., Optimal Control Problem for Low-Thrust Multiple Asteroid Tour Missions, Journal of Guidance, Control and Dynamics, Vol. 34, Issue 6, pp. 1709-1719, 2011. doi: 10.2514/1.53339

Price, K., Storn, R.M. \& Lampinen, J.A., Differential evolution: a practical approach to global optimization, Springer Science and Business Media, Berlin, Germany, 2006. doi: 10.1007/3-540-31306-0. 
Rayman, M. D. \& Mase, R. A., Dawn's exploration of Vesta, Acta Astronautica, Vol. 94, Issue 1, pp. 159-167, January-February 2014, doi: $10.1016 / j$.actaastro. 2013.08 .003

Russell, C. T., Raymond, C. A., Ammannito, E. et al., Dawn arrives at Ceres: Exploration of a small, volatile-rich world, Science, Vol. 353, Issue 6303, pp. 1008-1010, September 2016, doi: 10.1126/science.aaf4219

Sanchez Cuartielles, J. P., Gibbings, A., Snodgrass, C. \& Bowles, N., Asteroid belt multiple fly-by options for M-class missions, Proceedings of the 67th International Astronautical Congress, IAC-2016, 26-30 September 2016, Guadalajara, Mexico, https: //dspace.lib.cranfield.ac.uk/bitstream/1826/10874/1/Asteroid_ belt_multiple_flyby_options_for_M-Class_Missions-2016.pdf

Shang, H. \& Liu, Y., Assessing Accessibility of Main-Belt Asteroids Based on Gaussian Process Regression, Journal of Guidance, Control and Dynamics, Vol. 40, No. 5, pp. 1144-1154, May 2017, doi: 10.2514/1.G000576

Stuart, J. S., A Near-Earth Asteroid Population Estimated from the LINEAR Survey, Science, Vol. 294, Issue 5547, pp. 1691-1693, November 2001, doi: 10.1126/science. 1065318

Vallado, D., Fundamentals of Astrodynamics and Applications, Space Technology Library, Springer-Verlag, New York, USA, 2007

Vasile M., Minisci E., \& Locatelli M. An Inflationary Differential Evolution Algorithm for Space Trajectory Optimization. IEEE Transaction on Evolutionary Computation, Vol. 15, No. 2, pp. 267-281, April 2011. doi: 10.1109/TEVC. 2010.2087026

Vroom, A., Di Carlo, M., Romero Martin, J. M. \& Vasile, M., Optimal Trajectory Planning for Multiple Asteroid Tour Mission by Means of an Incremental Bio-Inspired Tree Search Algorithm, Proceedings of the 2016 IEEE Symposium Series on Computational Intelligence, 6-9 December 2016, Athens, Greece. doi: 10.1109/SSCI.2016.7850108

Wales, D.J. \& Doye, J.P., Global optimization by basin-hopping and the lowest energy structures of Lennard-Jones clusters containing up to 110 atoms, The Journal of Physical Chemistry A, Vol. 101, Issue 28, pp. 51115116, 1997. doi: 10.1021/jp970984n 
Yang, H. and Li, J. and Baoyin, H., Low-cost transfer between asteroids with distant orbits using mulitple gravity assists, Advances in Space Research, Vol. 56, Issue 5, pp. 837-848, 2015. doi: 10.1016/j .asr.2015.05.013

Zuiani, F., Vasile, M., Palmas, A. \& Avanzini, G. 2012, Direct transcription of low-thrust trajectories with finite trajectory elements, Acta Astronautica, Vol. 72, pp. 108-120, 2012. doi: 10.1016/j.actaastro.2011.09.011

Zuiani, F. \& Vasile, M., Extended analytical formulas for the perturbed Keplerian motion under a constant control acceleration, Celestial Mechanics and Dynamical Astronomy, Vol. 121, Issue 3, pp. 275-300, 2015. doi: $10.1007 / \mathrm{s} 10569-014-9600-5$ 